CERN-TH. 6827/93

PUPT-1382, LBL-33767, UCB-PTH-93/09, hep-th/9303046

\title{
Twists and Wilson Loops in the String Theory of Two Dimensional QCD ?
}

\author{
David J. Gross \\ Joseph Henry Laboratories, Princeton University \\ Theory Division \\ Princeton, New Jersey 08544, and \\ CERN, CH-1211 \\ gross@puhep1.princeton.edu \\ Geneva 23, Switzerland \\ Washington Taylor IV \\ Department of Physics, University of California \\ Theoretical Physics Group \\ Berkeley, California 94720 \\ and \\ Lawrence Berkeley Laboratory, \\ wati@physics.berkeley.edu \\ Berkeley, California 94720
}

\begin{abstract}
The string theory that describes two-dimensional QCD in an asymptotic $1 / N$ expansion is investigated further. A complete geometrical description of the QCD partition function on an arbitrary manifold is given in terms of maps of a two dimensional orientable surface onto the target space. This includes correction terms that arise on surfaces with genus $G \neq 1$, that are described geometrically by the insertion of extra "twist" points in the covering maps. In addition the formalism is derived for calculating the vacuum expectation value of an arbitrary product of Wilson loops on an arbitrary two dimensional manifold in terms of maps of an open string world sheet onto the target space.
\end{abstract}

CERN-TH. 6827/93

PUPT-1382，LBL-33767，UCB-PTH-93/09

March 1993

\footnotetext{
${ }^{*}$ This work was supported in part by the Director, Office of Energy Research, Office of High Energy and Nuclear Physics, Division of High Energy Physics of the U.S. Department of Energy under Contract DE-AC03-76SF00098 and in part by the National Science Foundation under grant PHY90-21984.
} 


\section{Introduction}

It has been known for some time that the two-dimensional quantum gauge theory is exactly soluble [1] and on a manifold $\mathcal{M}$ of genus $G$ and area $A$ has a partition function given by [2]

$$
\begin{aligned}
\mathcal{Z}_{\mathcal{M}} & =\int\left[\mathcal{D} A^{\mu}\right] e^{-\frac{1}{4 \tilde{g}^{2}} \int_{\mathcal{M}} d^{2} x \sqrt{g} \operatorname{Tr} F^{\mu \nu} F_{\mu \nu}} \\
& =\mathcal{Z}(G, \lambda A, N)=\sum_{R}(\operatorname{dim} R)^{2-2 G} e^{-\frac{\lambda A}{2 N} C_{2}(R)},
\end{aligned}
$$

where the sum is taken over all irreducible representations of the gauge group, with $\operatorname{dim} R$ and $C_{2}(R)$ being the dimension and quadratic Casimir of the representation $R$. ( $\lambda$ is related to the gauge coupling $\tilde{g}$ by $\lambda=\tilde{g}^{2} N$.) Throughout this paper, we will assume that the manifold $\mathcal{M}$ is endowed with a metric; however, the theory is invariant under area-preserving diffeomorphisms.

It was conjectured by one of us (DG) that the gauge theory with gauge group $S U(N)$ is equivalent to a string theory when one carries out an asymptotic expansion of (1.2) in powers of $1 / N$. The string coupling is identified with $1 / N$ and the string tension is given by $\lambda / 2[3]$. The strategy adopted to verify this conjecture was to explore whether the $1 / N$ expansion of $\mathcal{Z}_{\mathcal{M}}$ could calculated by summing over maps of a two-dimensional world sheet onto $\mathcal{M}$. The string theory would then be specified by the types of maps that would emerge and their weights in the sum. In a previous paper, we proved that this conjecture was correct [4 and identified the maps that reproduce the QCD partition function. When the target space is a torus, we were able to completely describe the partition function in terms of such a sum over maps; when the genus of the target space is other than one, there were extra terms in the partition function for which we had no geometric interpretation. In this paper we complete the discussion of the partition function on manifolds of arbitrary genus and extend the string theory to deal with Wilson loops.

Let us recall the results of [4]. Define $\Sigma_{G}$ to be the set of all continuous maps $\nu$ that map a (possibly disconnected) Riemann surface onto $\mathcal{M}$ such that the map $\nu$ is locally a covering map at all but a finite set of points of $\mathcal{M}$, with $n(\tilde{n})$ sheets whose orientation is the same as (opposite of) the orientation of $\mathcal{M}$. The asymptotic expansion of the partition function of the $S U(N)$ gauge theory on $\mathcal{M}$, in powers of $1 / N$, is then given by

$$
\mathcal{Z}(G, \lambda A, N)=\sum_{\nu \in \Sigma_{G}} \frac{(-1)^{\tilde{t}}}{\left|S_{\nu}\right|} e^{-\frac{(n+\tilde{n}) \lambda A}{2}} \frac{(\lambda A)^{(i+t+\tilde{t}+h)}}{i ! t ! \tilde{t} ! h !} N^{(n+\tilde{n})(2-2 G)-2(t+\tilde{t}+h)-i}\left[1+\mathcal{O}\left(\frac{1}{N}\right)\right]
$$


where $\left|S_{\nu}\right|$ is the order of symmetry of the map $\nu$ and $i, t, \tilde{t}, h$ are the quantities of each of the possible types of point singularities that can appear in the map. These singularities include branch points $(i)$, that can lie anywhere on $\mathcal{M}$; contracted tubes that connect sheets of the cover with the same $(t)$ and opposite $(\tilde{t})$ orientations and contracted handles $(h)$ of the covering space. The tubes and handles are mapped into points of $\mathcal{M}$ and are thus infinitesimal. The factor of $(\lambda A)^{(i+t+\tilde{t}+h)} / i ! t ! \tilde{t} ! h !$ corresponds to the range of possible locations of the singularity points in $\mathcal{M}$. The power of $1 / N$ is precisely $2 g-2$, where $g=1+(n+\tilde{n})(G-1)+t+\tilde{t}+h+i / 2$ is the genus of the covering space (the world sheet of the string). The $\mathcal{O}\left(\frac{1}{N}\right)$ corrections in this formula only occur when the genus of $\mathcal{M}$ is other than one.

An important feature of these maps is that they do not contain folds of non-zero length. This is an enormous simplification of the set of continuous maps between two-dimensional manifolds, and is the stringy reason, we believe, for the simplicity of $\mathrm{QCD}_{2}$. The suppression of folds means that all the maps in (1.3) have winding number greater than zero, since maps with winding number zero necessarily contain folds. This explains why our string theory, unlike any other, does not contain any propagating particles. It is clear that space-time histories that describe particle propagation correspond to maps with winding number zero. We must forbid such maps if we want to describe pure $\mathrm{QCD}_{2}$ which has no propagating states. Thus in our string theory even the center of mass degree of freedom, the so-called "tachyon", is absent. This bodes well for the analog string theory of QCD in higher dimensions, which remains to be constructed. Once the center of mass degree of freedom is absent in two dimensions there is no reason to expect a tachyon to appear in the higher dimensional theory. Presumably the dynamics of the string, which in two dimensions prevents folds that have infinite extrinsic curvature, will suppress extrinsic curvature in higher dimensions and eliminate the usual instabilities. Perhaps for the same reason graviton and dilaton modes will not appear. There is certainly no indication of them in our two dimensional theory. That our string theory does not contain the global gravitational and dilaton degrees of freedom found in Liouville string theories in two dimensions is indicated by the fact that it exists for any target space manifold and is insensitive to the metric of the target space. These features certainly befit a QCD string theory, which should not be a theory of gravity and should not contain either a dilaton or a tachyon.

Another feature of the string theory we are investigating is the existence of two separate chiral sectors, corresponding to covering spaces of two opposite orientations, as is appropriate 
for covering maps of a two dimensional manifold by a orientable world sheet. These sectors correspond in QCD to the contribution of quark-like representations of $S U(N)$ and their complex conjugates, anti-quark-like representations. These two sectors are coupled only by the infinitesimal tubes connecting sheets of opposite orientation.

The case when (1.3) is exact, that is when the target space is a torus, is the case of most physical interest; however, it is also useful to consider the theory for arbitrary genus. For genus $G \neq 1$ there are extra terms (and extra maps) that arise from the $1 / N$ corrections in the expansion of $(\operatorname{dim} R)^{2-2 G}$ in (1.2). In our previous work, these correction terms were not given a geometrical interpretation. In this paper, we describe the maps and extra factors that arise when the correction terms are included. We show that the extra terms can be described geometrically by the insertion of $|2-2 G|$ extra "twist" points in the covering maps. At these points, the sheets of each orientation are permuted in an arbitrary fashion by a singularity equivalent to a multiple branch point. Sheets of opposite orientation can also be connected at twist points by orientation-reversing tubes. When the sign of $I=2-2 G$ is positive, we refer to the twist points as " $\Omega$-points". The weights associated with this type of twist point are fairly simple. When the sign of $I$ is negative, however, the weights associated with the twist points are more complex; we refer to the twist points in this case as " $\Omega^{-1}$-points", since the effect of one of these twist points exactly cancels the effect of a single $\Omega$-point. We do not yet have an complete understanding of the significance of these points from the string theory perspective; however, it is hoped that their structure will give a useful clue towards discovering an action formalism for this string theory.

One of the other main results of this paper is the complete formulation of a method for calculating the vacuum expectation value of an arbitrary product of Wilson loops in a single chiral sector of the theory. In order to understand how the expectation value of a Wilson loop can be described as a sum over covering maps, it is necessary to define a set of observables for Wilson loops that is appropriate for the string theory. In the gauge theory picture, it is natural to associate each Wilson loop with a representation $R$ of the gauge group. The Wilson loop around a closed curve $\gamma$ is defined by

$$
W(R, \gamma)=\operatorname{Tr}_{R} \mathcal{P}\left\{\exp \left(i \oint_{\gamma} \mathbf{A}_{\mu} d x^{\mu}\right)\right\} \equiv \chi_{R}\left(U_{\gamma}\right)
$$

where the trace of the path-ordered exponential is taken in the representation $R$. In the string theory picture, we would like to associate the $1 / N$ expansion of expectation values of Wilson loops to the genus expansion of an open string theory, i.e. to maps of a two dimensional 
manifold with boundaries onto the target space on which there are certain specified curves (the closed paths that define the Wilson loops). These maps will map the boundaries of the string world sheet (namely certain circles $S^{1}$ ) onto the Wilson loops. Since our basic maps are covering maps we would expect that as we go around the Wilson loop $\gamma$ we will induce some permutation of the sheets covering the loop. We will therefore associate with each Wilson loop an integer $k$, and an element $\sigma$ of the permutation group $S_{k}$ on $k$ elements. Intuitively, $k$ will correspond to the number of sheets of the world sheet boundary covering the Wilson loop, and $S_{k}$ will be the permutation on those sheets that is realized by moving around the loop once. We thus want to define a different basis for the Wilson loops, labelled not by representations of $S U(N)$, but rather by elements of the symmetric group. If $\sigma$ is a permutation with cycles of size $k_{1}, \ldots, k_{s}$, the natural guess, whose validity we will establish, is that the appropriate gauge theory observable to pick out covering maps with boundary conditions described by $\sigma$ is

$$
\Upsilon_{\sigma}(U)=\prod_{j=1}^{s}\left(\operatorname{Tr} U^{k_{j}}\right),
$$

where the matrices $U$ are in the fundamental representation. The $\Upsilon$ observables are, like the characters, a complete set of class functions. In fact they are the well-known Schur functions of the eigenvalues of $U$ and are related to the characters by the Frobenius relations,

$$
\begin{aligned}
& \chi_{R}(U)=\sum_{\sigma \in S_{k}} \frac{\chi_{R}(\sigma)}{k !} \Upsilon_{\sigma}(U), \\
& \Upsilon_{\sigma}(U)=\sum_{R \in Y_{k}} \chi_{R}(\sigma) \chi_{R}(U),
\end{aligned}
$$

where $\chi_{R}(U)$ is the character of $U$ in the representation $R$ of $S U(N)$ associated with a Young tableau containing $k$ boxes, and $\chi_{R}(\sigma)$ is the character of the permutation $\sigma$ in the representation of $S_{k}$ associated with the same Young tableau. The sum in (1.7) is taken over all representations $R$ whose Young tableaux are in the set $Y_{k}$ of Young tableaux with $k$ boxes. Actually we will need to generalize the $\Upsilon_{\sigma}(U)$ basis and the Frobenius relations to account for the full set of relevant representations that contribute in the $1 / N$ expansion. The calculations relevant to this generalization are carried out in the Appendix. These relations are crucial in establishing the mathematical connection between the group theory of QCD and the geometry of the string theory.

The final result of our analysis of Wilson loops is that the vacuum expectation value of an arbitrary product of Wilson loops in a single sector of the theory can be described by a sum over covering maps similar to (1.3), where the boundary of the covering space is a union of 
covers of each Wilson loop, such that the boundary covering a Wilson loop $\gamma$ associated with a permutation $\sigma$ with $s$ cycles of length $k_{1}, \ldots, k_{s}$ is a disjoint union of $s$ loops that cover $\gamma$ exactly $k_{1}, \ldots, k_{s}$ times. Each of the regions into which the set of Wilson loops divides $\mathcal{M}$ is also associated with a number of $\Omega$-points or $\Omega^{-1}$-points. Although the sum over all regions of the number of $\Omega$-points must be $2-2 G$ (counting $\Omega^{-1}$-points as -1 ), these points are distributed according to the structure of the Wilson loops, so that even when $G=1$ it may be necessary to incorporate the $1 / N$ correction terms due to twist points. Thus, even to understand the theory on a torus it will be necessary to understand how the twists are related to the string theory action.

In the next section, we will review the results from [四 and describe how the corrections from twist points enter into the exact formula for the partition function for arbitrary genus in a single chiral sector of the theory. In Section 3 we discuss the exact partition function in the complete theory, including both chiral sectors. In order to deal with the most general relevant representations of $S U(N)$, we use the generalized Frobenius relations described in Appendix A. In Section 4, we derive expressions for the partition function on manifolds with boundaries, and describe how the partition function behaves when two manifolds are glued along a common boundary component. We use these results in section 5, where we derive the general formula for the vacuum expectation value of a product of Wilson loops in a single

chiral sector. In section 5, we also discuss the problem of calculating Wilson loops in the coupled theory. We present an algorithm that in principle produces the VEV of an arbitrary Wilson loop in the coupled theory, and describe some specific types of Wilson loops whose complete VEV's can easily be computed. In Section 6 we give a number of simple examples of the $1 / N$ expansions of the partition function as constructed by summing maps. In Section 7 we give some simple examples of Wilson loop vacuum expectation values. Section 8 contains an outline of how one can include dynamical quarks into the string picture. Finally we conclude with a summary of our results and discuss some further questions.

\section{The partition function}

The partition function for $\mathrm{QCD}_{2}$ (1.2) is expressed as a sum over all representations $R$ of the gauge group. Since the gauge group itself changes as we vary $N$, in order to construct an asymptotic expansion for the partition function in powers of $1 / N$, it is necessary to fix the representations so that the dimension and quadratic Casimir of a representation 
can themselves be expanded in powers of $1 / N$. This can be accomplished by the use of Young tableaux to label the representations. Every representation $R$ is associated with a Young tableau containing some number of boxes $n$, that are distributed in rows of length $n_{1} \geq n_{2} \cdots \geq n_{l}>0$ and columns of length $c_{1} \geq c_{2} \cdots \geq c_{k}>0$, where $k=n_{1}$, and $l=c_{1}$. We will abuse notation slightly and sometimes use $R$ to refer to the Young tableau as well as to the representation itself. The quadratic Casimir of the representation $R$ is given by

$$
C_{2}(R)=n N+\tilde{C}(R)-\frac{n^{2}}{N}
$$

where

$$
\tilde{C}(R)=\sum_{i} n_{i}^{2}-\sum_{i} c_{i}^{2}
$$

It was shown in 4 that the term $\tilde{C}(R)$ is related to a character of the permutation group by

$$
\tilde{C}(R)=\frac{n(n-1) \chi_{R}\left(T_{2}\right)}{d_{R}},
$$

where $d_{R}=\chi_{R}(1)$ is the dimension of the representation of the permutation group $S_{n}$ associated with the Young tableau $R$, and $\chi_{R}\left(T_{2}\right)$ is the character in this representation of any permutation in the conjugacy class $T_{2}$ of permutations containing a single cycle of length 2 and $n-2$ cycles of length 1 .

The dimension of the representation $R$ of $S U(N)$ can be expressed in a form appropriate for the large $N$ expansion by setting $U=1$ in the Frobenius formula (1.6),

$$
\operatorname{dim} R=\frac{1}{n !} \sum_{\sigma \in S_{n}} N^{K_{\sigma}} \chi_{R}(\sigma),
$$

where $K_{\sigma}$ is the number of cycles in the permutation $\sigma$. Note that the leading order term in this polynomial arises from the identity permutation $\left(\sigma=\left[1^{n}\right]\right)$ and is equal to $d_{R} N^{n} / n$ !. By summing over all $n$, and for each $n$ summing over all Young tableaux in $Y_{n}$, we can write (ए.2) in a form that is appropriate for an asymptotic expansion of the partition function in powers of $1 / N$, namely

$$
Z(G, \lambda A, N)=\sum_{n=0}^{\infty} \sum_{R \in Y_{n}}(\operatorname{dim} R)^{2-2 G} e^{-\frac{\lambda A}{2 N} C_{2}(R)}
$$

The idea is to carry out the $1 / N$ expansion of $C_{2}(R)$ and of $\operatorname{dim}(R)$, using (2.1) and (2.4), inside the sum over the number of boxes $n$. 
This expansion, however, only contains half of the full theory. The representations associated with Young tableaux with a fixed number of boxes arise from symmetrizing and antisymmetrizing tensor products of the fundamental representation of $S U(N)$. It is also necessary to include representations that arise from tensor products of the conjugate representation $U \rightarrow U^{\dagger}$; these representations also have quadratic Casimirs with leading terms of order $N$. The most general representation that we must consider is a composite representation that we get by taking the largest irreducible representation contained in the tensor product of a representation $R$ with a finite number of boxes, and the conjugate $\bar{S}$ of another representation $S$, that also has a finite number of boxes. Specifically, given two representations $R$ and $S$, whose Young tableaux contain $n$ and $\tilde{n}$ boxes respectively, with rows of length $n_{i}$ and $\tilde{n}_{i}$, and columns of length $c_{i}$ and $\tilde{c}_{i}$, we can form a new representation $\bar{S} R$, with column lengths

$$
\left\{\begin{array}{ll}
N-\tilde{c}_{\tilde{n}_{1}+1-i}, & i \leq \tilde{n}_{1} \\
c_{i-\tilde{n}_{1}}, & i>\tilde{n}_{1}
\end{array}\right\}
$$

The quadratic Casimir of the representation $\bar{S} R$ is given by

$$
C_{2}(\bar{S} R)=C_{2}(R)+C_{2}(S)+\frac{2 n \tilde{n}}{N} .
$$

It can also be shown that the dimension of the representation $\bar{S} R$ is given by

$$
\begin{aligned}
\operatorname{dim} \bar{S} R & =\operatorname{dim} S \operatorname{dim} R \prod_{i, j} \frac{(N+1-i-j)\left(N+1-i-j+n_{i}+\tilde{n}_{j}\right)}{\left(N+1-i-j+n_{i}\right)\left(N+1-i-j+\tilde{n}_{j}\right)} \\
& =\operatorname{dim} S \operatorname{dim} R+\mathcal{O}\left(\frac{1}{N^{2}}\right) .
\end{aligned}
$$

Using the results of Appendix A we shall present a more useful formula for the $\frac{1}{N^{2}}$ corrections to $\operatorname{dim} \bar{S} R$ in the next section.

By including all composite representations, which are the only representations of $S U(N)$ whose quadratic Casimir grows like $N$, we can write the partition function of the full gauge theory as

$$
\mathcal{Z}(G, \lambda A, N)=\sum_{n} \sum_{\tilde{n}} \sum_{R \in Y_{n}} \sum_{S \in Y_{\tilde{n}}}(\operatorname{dim} \bar{S} R)^{2-2 G} e^{-\frac{\lambda A}{2 N}\left[C_{2}(R)+C_{2}(S)+\frac{2 n \tilde{n}}{N}\right]},
$$

and derive the asymptotic expansion by expanding the Casimirs and dimensions in powers of $1 / N$ inside the sum. The corrections to this expansion are then of order $\exp [-N]$, corresponding to non-perturbative string corrections of order $\exp \left[-1 / g_{\text {string }}\right]$, that are invisible in string perturbation theory. 
This partition function can be factored into two copies of the partition function (2.5), that are only coupled by the term $\frac{2 n \tilde{n}}{N}$ in the quadratic Casimir and by some terms of order $1 / N^{2}$ that arise from the non-leading terms in the expansion of the dimension. We refer to the two factors in this product as chiral sectors of the theory. We interpret the two chiral sectors as corresponding to orientation-preserving and to orientation-reversing string maps; under this interpretation, the coupling terms in the exponential correspond to infinitesimal orientation-reversing tubes that connect covering sheets of opposite relative orientation.

To understand how (2.10) can be understood in terms of a string theory, it is simplest to analyze a single sector of the theory first, and then to describe how the sectors are coupled. Therefore, let us now consider a single chiral sector of the theory, with partition function (2.5). Breaking up the term $n^{2} / N$ from the quadratic Casimir into a sum of terms $n / N+n(n-1) / N$ [5], and expanding the exponential of the quadratic Casimir, this partition function, which we denote by $Z^{+}$, can be rewritten as

$$
\begin{array}{r}
Z^{+}(G, \lambda A, N)=\sum_{n=0}^{\infty} \sum_{R \in Y_{n}} e^{-\frac{n \lambda A}{2}} \sum_{i, t, h} \frac{(\lambda A)^{i+t+h}}{i ! t ! h !} \frac{(-1)^{i} n^{h}\left(n^{2}-n\right)^{t+i}}{(n !)^{2-2 G} 2^{i+t+h} d_{R}^{i}}\left(\chi_{R}\left(T_{2}\right)\right)^{i} \\
\cdot N^{n(2-2 G)-i-2(t+h)}\left(\sum_{\sigma \in S_{n}} \frac{\chi_{R}(\sigma)}{N^{n-K_{\sigma}}}\right)^{2-2 G} .
\end{array}
$$

In order to rewrite (2.11) in terms of a sum over maps, it will be useful to recall some elementary results from the representation theory of the symmetric group $S_{n}$. One result we will use repeatedly is that when $T$ is a conjugacy class of elements in $S_{n}$ (i.e. $\sigma^{\prime} \in T_{\sigma}$ if $\sigma=s \sigma s^{-1}$ for $s \in S_{n}$ ), and $\rho$ is an arbitrary element of $S_{n}$, the characters of $T$ and $\rho$ in a fixed representation $R$ can be combined according to the formula

$$
\sum_{\sigma \in T} \frac{\chi_{R}(\sigma)}{d_{R}} \chi_{R}(\rho)=\sum_{\sigma \in T} \chi_{R}(\sigma \rho)
$$

This result follows immediately from the fact that in any representation $R$ the sum of the matrices associated with the elements in $T$ must be proportional to the identity matrix, since this sum commutes with all elements of $S_{n}$

Associated with the symmetric group, as with any finite group, there is a natural group algebra, consisting of all linear combinations of group elements with real coefficients. Elements of the group algebra are added and multiplied in the natural way. A Kronecker-type delta function can be defined on the symmetric group algebra; if $\sum_{\sigma} c_{\sigma} \sigma$ is an element of the 
group algebra, then we define the delta function by

$$
\delta\left(\sum_{\sigma} c_{\sigma} \sigma\right)=c_{1},
$$

where $c_{1}$ is the coefficient of the identity permutation.

Other results from the representation theory of $S_{n}$ that we will need are

$$
\delta(\rho)=\frac{1}{n !} \sum_{R} d_{R} \chi_{R}(\rho),
$$

which follows from the completeness of the characters, and the relation

$$
\left(\frac{n !}{d_{R}}\right)^{2}=\sum_{s, t \in S_{n}} \frac{\chi_{R}\left(s t s^{-1} t^{-1}\right)}{d_{R}},
$$

that may be proven using (2.12).

Finally there is an element $\Omega_{n}$ of the group algebra on $S_{n}$, defined by (recall that $K_{\sigma}=$ number of cycles of $\sigma$ ),

$$
\Omega_{n}=\sum_{\sigma \in S_{n}} \sigma N^{K_{\sigma}-n},
$$

that will be useful in understanding the effects of the dimension term in the partition function. In fact, the formula for the dimension (2.4) can be written as

$$
\operatorname{dim} R=\frac{N^{n}}{n !} \chi_{R}\left(\Omega_{n}\right)
$$

Since we will also encounter inverse powers of the dimension it is useful to invert $\Omega_{n}$. Since $\Omega_{n}$ is a polynomial in $1 / N$, with a leading term of 1 times the identity permutation (in fact $\delta\left(\Omega_{n}\right)=1$ ), we can write

$$
\Omega_{n}=1+\tilde{\Omega}_{n}
$$

where $\tilde{\Omega}_{n}$ is of order $1 / N . \Omega_{n}$ has a formal inverse $\Omega_{n}^{-1} . \Omega_{n}^{-1}$ is an infinite series in $1 / N$, that also has a leading term of 1 times the identity permutation,

$$
\begin{aligned}
\Omega_{n}^{-1} & =\sum_{\sigma} \omega_{\sigma} \sigma=\sum_{k=0}^{\infty}(-1)^{k} \tilde{\Omega}_{n}^{k} \\
& =\mathbf{1}\left(1+O\left(\frac{1}{N^{2}}\right)\right)-\frac{1}{N} \sum_{\sigma \in T_{2}} \sigma\left(1+O\left(\frac{1}{N}\right)\right)+\cdots
\end{aligned}
$$

Since in any representation $R, \Omega_{n}$ is proportional to the identity matrix, it follows that

$$
\frac{1}{\chi_{R}\left(\Omega_{n}\right)}=\frac{1}{d_{R}^{2}} \chi_{R}\left(\Omega_{n}^{-1}\right) .
$$


Thus, we can write

$$
\frac{1}{\operatorname{dim} R}=\frac{n !}{N^{n} d_{R}^{2}} \chi_{R}\left(\Omega_{n}^{-1}\right)
$$

More generally,

$$
(\operatorname{dim} R)^{m}=\left(\frac{N^{n} d_{R}}{n !}\right)^{m} \frac{\chi_{R}\left(\Omega_{n}^{m}\right)}{d_{R}}
$$

for both positive and negative $m$.

From equations (2.12), (2.14), (2.15), and (2.17), the asymptotic expansion (2.11) can be rewritten in the form

$$
\begin{aligned}
Z^{+}(G, \lambda A, N)= & \sum_{n, i, t, h} e^{-\frac{n \lambda A}{2}} \frac{(\lambda A)^{i+t+h}}{i ! t ! h !} N^{n(2-2 G)-i-2(t+h)} \frac{(-1)^{i} n^{h}\left(n^{2}-n\right)^{t}}{2^{t+h}} \\
& \cdot \sum_{p_{1}, \ldots, p_{i} \in T_{2}} \sum_{s_{1}, t_{1}, \ldots, s_{G}, t_{G} \in S_{n}}\left[\frac{1}{n !} \delta\left(p_{1} \cdots p_{i} \Omega_{n}^{2-2 G} \prod_{j=1}^{G} s_{j} t_{j} s_{j}^{-1} t_{j}^{-1}\right)\right]
\end{aligned}
$$

This equation is derived from (2.11) by applying (2.15) $G$ times, repeatedly applying (2.12) until all characters are combined into a single character, and then applying (2.14) to turn the sum over representations into a single delta function.

It is now possible to give a geometric interpretation to the partition function by showing that (2.24) is equal to a sum over covering maps of $\mathcal{M}$. The most unusual part of this interpretation is the effect of the $\Omega_{n}$ terms in the delta function. These terms correspond to having extra twists in the covering at precisely $|I|=|2-2 G|$ points in $\mathcal{M}$. To be more specific, let us choose an arbitrary set of points $z_{1}, \ldots, z_{|I|}$ in $\mathcal{M}$. For a fixed value of $i$, we also select a set of branch points $w_{1}, \ldots, w_{i} \in \mathcal{M}$. For the moment, let us assume that $G \leq 1$ so $I$ is positive. In this case, we assert that for a fixed value of $n$, the expression

$$
\sum_{p_{1}, \ldots, p_{i} \in T_{2}} \sum_{s_{1}, t_{1}, \ldots, s_{G}, t_{G} \in S_{n}} N^{n(2-2 G)-i}\left[\frac{1}{n !} \delta\left(p_{1} \cdots p_{i} \Omega_{n}^{2-2 G} \prod_{j} s_{j} t_{j} s_{j}^{-1} t_{j}^{-1}\right)\right]
$$

is precisely equal to the sum of a factor $N^{2-2 g} /\left|S_{\nu}\right|$ over all $n$-fold covers $\nu$ of $\mathcal{M}$ that have single branch points at each of the points $w_{1}, \ldots, w_{i}$, and that have arbitrary twists (permutations of sheets) arising from multiple branch points at $z_{1}, \ldots, z_{|I|}$, where $g$ is the genus of the covering space, and $\left|S_{\nu}\right|$ is the symmetry factor of the cover (the number of homeomorphisms $\pi$ of the covering space that satisfy $\nu \pi=\nu$ ).

This assertion can be proven by considering the fundamental group of the manifold $\mathcal{M}$ with punctures at the points $z_{j}, w_{j}, \mathcal{G}=\pi_{1}\left(\mathcal{M} \backslash\left\{z_{1}, \ldots, z_{|I|}, w_{1}, \ldots, w_{i}\right\}\right)$. This group is generated by a set of loops $a_{1}, b_{1}, \ldots, a_{G}, b_{G}$ that are a basis for the homology of $\mathcal{M}$, a set 
of loops $c_{1}, \ldots, c_{i}$ around the branch points, and a set of loops $d_{1}, \ldots, d_{|I|}$ around the twist points. The only relation satisfied by these generators is

$$
c_{1} \cdots c_{i} d_{1} \cdots d_{|I|} a_{1} b_{1} a_{1}^{-1} b_{1}^{-1} a_{2} b_{2} a_{2}^{-1} b_{2}^{-1} \cdots a_{G} b_{G} a_{G}^{-1} b_{G}^{-1}=1
$$

Assign a set of labels $1, \ldots, n$ to the sheets of a covering space at a fixed basepoint. As one transverses the manifold around any loop that is not homotopically trivial the labeling of the sheets is permuted. We will define the permutations on the covering sheet labels that are realized by the loops $a_{j}, b_{j}, c_{j}, d_{j}$ to be elements of the permutation group of $n$ objects denoted by $s_{j}, t_{j}, p_{j}, q_{j}$ respectively. We see then that each cover corresponds to a homomorphism $H$ from $\mathcal{G}$ into the symmetric group $S_{n}$. Note that the branch points must be associated with permutations in the conjugacy class $T_{2}$, since they are assumed to be branch points with branching number 1 . Any two homomorphisms $H$ and $H^{\prime}$ that are related through conjugation by an element of the symmetric group, $H^{\prime}=\rho H \rho^{-1}$, correspond to distinct labelings of a single covering space. Every symmetry of the covering space corresponds to a permutation that leaves $H$ invariant. Since there are exactly $n$ ! possible labelings of a given cover, in order to sum over all covers a factor of $1 /\left|S_{\nu}\right|$, it will suffice to sum over all homomorphisms $H$ a factor of $1 / n$ !. Writing the sum of $N^{2-2 g} / n$ ! over all homomorphisms gives exactly the sum in (2.25), where each branch point contributes -1 to the Euler characteristic $2-2 g$ of the covering space, and where each twist $q_{j}$ contributes $K_{q_{j}}-n$. For a more detailed calculation of this type, see [4].

We can thus rewrite the partition function (2.24) completely in terms of a sum over covers when $G \leq 1$. We define the set of orientation-preserving covers of $\mathcal{M}$ with $|I|$ twist points to be $\Sigma_{+}(\mathcal{M})$. We include in this set all maps from a Riemann surface $\mathcal{M}_{g}$ of genus $g$ onto $\mathcal{M}$ that are orientation-preserving maps, and that are covering maps in a local neighborhood of all points in $\mathcal{M}$ except for a finite number of singular points. The singular points are divided into $|I|$ twist points, $i$ branch points, $t$ points where loops in $\mathcal{M}_{g}$ are mapped to single points in $\mathcal{M}$ (orientation-preserving tubes), and $h$ points where entire handles of $\mathcal{M}_{g}$ are mapped to single points in $\mathcal{M}$. The twist points are fixed points $z_{j}$ in $\mathcal{M}$ where the covering map takes a set of neighborhoods in $\mathcal{M}_{g}$ into a single neighborhood of $z_{j}$, such that the preimage of $z_{j}$ in each of the neighborhoods in $\mathcal{M}_{g}$ is a single point. These points can be viewed as points where multiple single branch points have combined to give an arbitrary permutation of the sheets of the covering space, without adding handles or tubes.

The set $\Sigma_{+}(\mathcal{M})$ can be separated into disjoint components labeled by the integers $i, t, h$. Each of these components breaks down further into a set of disjoint connected components 
labeled by topological type, each of which is a continuous manifold parameterized by the positions of the branch points $w_{j}$, and the positions of the tubes and handles, $x_{j}$ and $y_{j}$. On each connected component of $\Sigma_{+}(\mathcal{M})$, we can define an integration measure $d \nu$ for the parameters $w_{j}, x_{j}$, and $y_{j}$ that is proportional to a product of the area measures for each of the parameters. Since the branch points, tubes, and handles are indistinguishable, by integrating over the parameters we get a factor of $(\lambda A)^{(i+t+h)} / i ! t ! h$ ! when the proportionality constant is $\lambda$. We define an integral over all of $\Sigma_{+}(\mathcal{M})$ to be a sum over all connected components of $\Sigma_{+}(\mathcal{M})$ of the integral with measure $d \nu$ over each connected component. In terms of such an integral, the partition function (2.24) is simply given by

$$
Z^{+}(G, \lambda A, N)=\int_{\Sigma_{+}(\mathcal{M})} d \nu e^{-\frac{n \lambda A}{2}} \frac{(-1)^{i} N^{2-2 g}}{\left|S_{\nu}\right|}
$$

where $n$ is the winding number of the map $\nu, i$ is the number of branch points in $\nu,\left|S_{\nu}\right|$ is the symmetry factor, and $g$ is the genus of the covering space. The extra factors of $n / 2$ and $n(n-1) / 2$ in (2.24) appear because a handle can be mapped onto any of the $n$ sheets of the cover and has an extra symmetry factor of 2 , and a tube can connect an arbitrary pair of the sheets.

We have now given a simple formula for the partition function on a manifold with genus $G \leq 1$ in terms of a sum over covering maps. It is not hard to see how this formula must be modified if the genus is larger than 1 . When $I$ is positive, the algebra element $\Omega_{n}$ appears in the expression for the partition function to a positive power. Each instance of $\Omega_{n}$ in the delta function in (2.24) corresponds to a twist point in $\mathcal{M}$ where all twists $q_{j}$ are given a weight of $N^{K_{q_{j}}-n}$. We will refer to points of this type as " $\Omega$-points". For instance, to calculate the partition function on a sphere, one must sum over all covers with two $\Omega$-points. When $I$ is negative, the number of factors of $\Omega_{n}$ in the delta function is negative. This can be interpreted as indicating that we must sum over covers that contain twist points with a different set of weights. We will refer to the $-I$ twist points of this new type as " $\Omega^{-1}$-points". If we write the coefficients of the algebra element $\Omega_{n}^{-1}$ as $\omega_{\sigma}$, as in (2.19), then the weight of an $\Omega^{-1}$-point with a permutation $q_{j}$ will be $\omega_{q_{j}}$. The coefficients $\omega_{\sigma}$ are infinite series in $1 / N$. A simpler way to interpret these $\Omega^{-1}$-points is to use the expression (2.19) for $\Omega_{n}^{-1}$ in terms of $\tilde{\Omega}_{n}$. From this expression, it is clear that an $\Omega^{-1}$-point can be viewed as a combination of an arbitrary number $(x \geq 0)$ of nontrivial twists such as would arise at an $\Omega$-point, with each twist carrying a factor of -1 . An $\Omega^{-1}$-point is then a point where an arbitrary number of nontrivial twists coalesce to form a singularity with an arbitrary permutation on the sheets, 
and possibly internal handles or tubes. By using the same logic as in the case $G \leq 1$, the asymptotic expansion for a single chiral sector of the gauge theory on a manifold with an arbitrary genus $G$ can be written as

$$
Z^{+}(G, \lambda A, N)=\int_{\Sigma_{+}(\mathcal{M})} d \nu e^{-\frac{n \lambda A}{2}} \frac{(-1)^{i} N^{2-2 g}}{\left|S_{\nu}\right|} \prod_{j=1}^{\max (0,-I)}(-1)^{x_{j}},
$$

where $x_{j}$ are the numbers of nontrivial twists at the $\max (0,-I) \Omega^{-1}$-points in the map $\nu$, and $n, i,\left|S_{\nu}\right|$ and $g$ are as above.

Although the introduction of twist points ( $\Omega$-points and $\Omega^{-1}$-points) gives us a geometric interpretation of all terms in the partition function for the theory on an arbitrary genus Riemann surface, it is rather unclear how these points might be described by a string theory action. Since locations of the twist points are not integrated over (the twist points do not carry factors of the area), they seem to correspond to some global, rather than local structure. In fact, although we have described the twists as occurring at fixed points in the manifold $\mathcal{M}$, they may in fact describe some purely non-local structure. Because the number of $\Omega$-points is equal to the Euler characteristic of the manifold $\mathcal{M}$, it is tempting to speculate that these twist points are somehow related to the divisor of the tangent bundle of $\mathcal{M}$. One might hope to find a suitable way of coupling fermions to the theory in a way that would simultaneously cancel folds and give rise to terms like those coming from $\Omega$-points and $\Omega^{-1}$-points; however, we have not yet succeeded in finding a string action that has all these desired properties.

\section{The Complete Theory}

The analysis performed in the previous section applied only to a single chiral sector of the theory. It is possible to combine the two chiral sectors, and to use a similar formalism in describing the partition function for the complete theory. When we combine the two sectors, we can consider the theory to include independent sums over all covering maps that are orientation-preserving and orientation-reversing. These sets of covering maps are coupled. The simplest coupling arises from the extra term $2 n \tilde{n} / N$ in the quadratic Casimir (2.7). It has a natural interpretation as corresponding to an arbitrary number of orientation-reversing tubes connecting sheets of covers from the two sectors. These tubes are mapped onto single points of the target space $\mathcal{M}$. The positions of these tubes are integrated over just as are the positions of orientation-preserving tubes in the two sectors. Thus each tube contributes 
a factor of $n \tilde{n} \lambda A / N^{2}$, since the genus of the map is increased by one for each additional tube. In addition it appears that we must include a minus sign each time such a tube occurs. The contribution of the orientation reversing tubes then exponentiates to yield a factor of $\exp \left[-(\lambda A / 2) 2 n \tilde{n} / N^{2}\right]$ in the partition function, reproducing the coupling term in the exponent in (2.10).

There are also extra terms in the formula for the dimension of a composite representation (2.9) that we argue correspond to additional tubes connecting the two sectors. To derive these we need to determine the $1 / N$ expansion of the dimension of the composite representation $\bar{S} R$. This analysis is carried out in Appendix A, where we generalize the Frobenius relations for the characters of composite representations of $S U(N)$ and derive the $1 / N$ expansion of the dimensions of these representations. The formula (A.25) derived there can be written as

$$
\operatorname{dim} \bar{S} R=\frac{1}{n ! \tilde{n} !} \sum_{\sigma, \tau} N^{K_{\sigma}+K_{\tau}} \chi_{R}(\sigma) \chi_{R}(\tau)\left(\sum_{t, j_{u}, \tilde{j}_{u}} \prod_{u=1}^{t}\left(-\frac{k_{j_{u}}}{N^{2}}\right)\right)
$$

where $k_{j}, \tilde{k}_{j}$ are the lengths of the cycles of $\sigma, \tau$ respectively. The sum is taken over all disjoint pairs $\left(j_{u}, \tilde{j}_{u}\right)$ of cycles of $\sigma$ and $\tau$ that are of the same length. This means that we sum over all $j_{u}$ and $\tilde{j}_{u}$ such that for $u \neq u^{\prime}, j_{u} \neq j_{u^{\prime}}$ and $\tilde{j}_{u} \neq \tilde{j}_{u^{\prime}}$, and such that for all $u$, $k_{j_{u}}=\tilde{k}_{\tilde{j}_{u}}$.

Using this formula we can go through the same steps as before to write the complete partition function as a sum over maps. We define a more general object $\Omega_{n \tilde{n}}$ to be an element of the algebra of the tensor product group $S_{n} \otimes S_{\tilde{n}}$, given by

$$
\Omega_{n \tilde{n}}=\sum_{\sigma, \tau} \sigma \otimes \tau \sum_{v, v^{\prime}}(-1)^{K_{v}} C_{v} N^{K_{\sigma \backslash v}+K_{\tau \backslash v}-n-\tilde{n}}
$$

Similar to the case of a single sector, we have

$$
\operatorname{dim} \bar{S} R=\chi_{R \otimes S}\left(\Omega_{n \tilde{n}}\right)
$$

Again, $\Omega_{n \tilde{n}}$ is a polynomial in $1 / N$ with leading term 1 , and can be written

$$
\Omega_{n \tilde{n}}=1+\tilde{\Omega}_{n \tilde{n}}
$$

We can expand $\Omega_{n \tilde{n}}^{-1}$ as a power series in $1 / N$, and write

$$
\Omega_{n \tilde{n}}^{-1}=\sum_{x=0}^{\infty}(-1)^{x} \tilde{\Omega}_{n \tilde{n}}
$$


The partition function in the complete theory on an arbitrary genus surface can now be written as a sum over maps,

$$
\mathcal{Z}(G, \lambda A, N)=\int_{\Sigma(\mathcal{M})} d \nu e^{-\frac{(n+\tilde{n}) \lambda A}{2}} \frac{(-1)^{i+\tilde{t}} N^{2-2 g}}{\left|S_{\nu}\right|} \prod_{j=1}^{\max (0,-I)}(-1)^{x_{j}}
$$

where $\Sigma(\mathcal{M})$ is the set of all covering spaces of $\mathcal{M}$ with a finite number of singular points, $\tilde{t}$ orientation-reversing tubes, and $2-2 G \Omega$-points. In the coupled theory an $\Omega$-point is a point at which in each sector the sheets can be twisted arbitrarily, and where there can also be orientation-reversing tubes connecting cycles of these twists, subject to the conditions that no cycle can be attached to more than one tube, and tubes can only connect cycles of the same order. (These conditions on the placement of tubes follow from (3.2).) As usual, these orientation-reversing tubes carry a factor of -1 . In the coupled theory, $\Omega^{-1}$-points again may consist of an arbitrary number of nontrivial twists, each carrying a factor of -1 , where among nontrivial twists we include all possible singularities that might occur at an $\Omega$-point, including the case where the permutations in both sectors are trivial but there is at least one tube connecting sheets of opposite orientation (cycles of length one).

This completes the derivation of the string theory representation, written as a sum over maps, for the two-dimensional QCD partition function, to all orders in $1 / N$ and on an arbitrary space-time background. Except for the $\Omega$-points all the features of the maps are very simple - the maps are simply minimal area maps with no folds, consisting of covers of the target space with possible branchpoints, contracted handles and tubes mapped to a point. The counting of the maps is the natural topological counting. On surfaces of genus $G \neq 1$ there are in addition the extra maps with twists and tubes connecting the twist points at $|2-2 G|$ fixed points on the manifold. The challenge is to find a string Lagrangian whose partition function yields this set of maps.

\section{Gluing manifolds}

In this section, we will calculate the partition function of $\mathrm{QCD}_{2}$ on manifolds with boundaries in terms of a sum over string maps, and derive a set of formulae for how these partition functions combine when manifolds are glued together along a common boundary region. These results will be used in the next section to calculate vacuum expectation values of Wilson loops. The equations in this section can also be used to describe the $\mathrm{QCD}_{2}$ string 
theory in a more geometric fashion, by gluing together the fundamental objects described by a sphere with 1,2 , and 3 boundary components (caps, propagators, and vertices). In this section, as in the next, we will concentrate on a single sector of the theory; however, we give generalizations of all results to the coupled theory with the exception of the gluing rule along an edge segment (which is not used in the Feynman diagram calculations). An analogous gluing equation can be derived in the coupled theory; however, we do not have a simple closed-form expression for the result, which leads to rather complicated structures in Wilson loop calculations in the full theory.

First, we will need a formula for the partition function on a (compact) Riemann surface $\mathcal{M}$ of genus $G$ and area $A$ with an arbitrary boundary. Assume that the manifold $\mathcal{M}$ has a boundary $\partial \mathcal{M}$ that is a disjoint union of $b$ copies of the circle $S^{1}$. We denote these components of the boundary by $E_{1}, \ldots, E_{b}$. To calculate the partition function on such a manifold we must specify boundary conditions on the gauge fields. The holonomies of the gauge field around the boundary curves are given by

$$
U_{j}=\mathcal{P}\left\{\exp \left(i \oint_{E_{j}} \mathbf{A}_{\mu} d x^{\mu}\right)\right\}
$$

where the path-ordered exponential is again taken in the fundamental representation. When the holonomies around the boundary curves are taken to be fixed values $U_{1}, \ldots U_{b}$, the partition function can be calculated in a similar fashion to the partition function on a closed manifold, and is given by [1, 6]

$$
\mathcal{Z}\left(G, \lambda A, N ;\left\{U_{1}, \ldots, U_{b}\right\}\right)=\sum_{R}(\operatorname{dim} R)^{2-2 G-b}\left(\prod_{j=1}^{b} \chi_{R}\left(U_{j}\right)\right) e^{-\frac{\lambda A}{2 N} C_{2}(R)} .
$$

In a single sector of the theory, we can use the Frobenius relations (1.6) to express the partition function (4.2) in terms of the class functions $\Upsilon_{\sigma}(U)$,

$$
\begin{aligned}
Z^{+}\left(G, \lambda A, N ;\left\{U_{i}\right\}\right) & =\sum_{n} \sum_{R \in Y_{n}}(\operatorname{dim} R)^{2-2 G-b} e^{-\frac{\lambda A}{2 N} C_{2}(R)} \prod_{j=1}^{b} \chi_{R}\left(U_{j}\right) \\
& =\sum_{n} \sum_{R \in Y_{n}}(\operatorname{dim} R)^{2-2 G-b} e^{-\frac{\lambda A}{2 N} C_{2}(R)} \prod_{j=1}^{b}\left(\sum_{\sigma_{j}} \frac{\chi_{R}\left(\sigma_{j}\right)}{n !} \Upsilon_{\sigma_{j}}\left(U_{j}\right)\right)
\end{aligned}
$$

By an argument essentially identical to that of the previous section, this expression can be rewritten as a sum/integral over the set $\Sigma_{+}(\mathrm{M})$ of orientation-preserving covers (with 
$2-2 G-b \Omega$-points and a world-sheet with boundary) of $\mathcal{M}$,

$$
Z^{+}\left(G, \lambda A, N ;\left\{U_{i}\right\}\right)=\int_{\Sigma_{+}(\mathcal{M})} d \nu e^{-\frac{n \lambda A}{2}} \frac{(-1)^{i} N^{2-2 g-\beta}}{\left|S_{\nu}\right|}\left(\prod_{j=1}^{\max (0,-I)}(-1)^{x_{j}}\right)\left(\prod_{k=1}^{b} \Upsilon_{\sigma_{k}}\left(U_{k}\right)\right)
$$

where the measure $d \nu$ is defined as in section $2, \beta=\sum K_{\sigma_{k}}$ is the number of boundary components of the covering space, and where $\sigma_{k}$ is in the conjugacy class of the permutation on sheets of the cover described by traversing the boundary component $E_{k}$ of $\mathcal{M}$.

A formula that more precisely captures the geometrical nature of this partition function is found by evaluating the expectation value of $\prod_{i=1}^{b} \Upsilon_{\sigma_{i}}\left(U_{i}^{\dagger}\right)$. This then yields

$$
\begin{aligned}
Z^{+}\left(G, \lambda A, N ;\left\{\sigma_{i}\right\}\right) & =<\prod_{i=1}^{b} \Upsilon_{\sigma_{i}}\left(U_{i}^{\dagger}\right)> \\
& =\int_{\Sigma_{+}\left(\mathcal{M} ;\left\{\sigma_{i}\right\}\right)} d \nu e^{-\frac{n \lambda A}{2}} \frac{(-1)^{i} N^{2-2 g-\beta}}{\left|S_{\nu}\right|}\left(\prod_{j=1}^{\max (0,-I)}(-1)^{x_{j}}\right),
\end{aligned}
$$

where the condition on the maps $\nu$ in $\Sigma_{+}\left(\mathcal{M} ;\left\{\sigma_{i}\right\}\right)$ is that when we transverse the boundary component $E_{i}$ the sheets of the cover undergo a permutation in the class of $\sigma_{i}$.

It is straightforward to generalize (4.5) to the coupled theory. By using the generalized Frobenius relations from Appendix A, the character of an arbitrary composite representation can be rewritten as a sum of generalized $\Upsilon$ functions. By an identical analysis to that performed above, we find that the expectation value of a product $\prod_{i=1}^{b} \Upsilon_{\bar{\sigma}_{i} \tau_{i}}\left(U_{i}, U_{i}^{\dagger}\right)$ is given by the sum over maps

$$
\begin{aligned}
\mathcal{Z}\left(G, \lambda A, N ;\left\{\sigma_{i}, \tau_{i}\right\}\right) & =<\prod_{i=1}^{b} \Upsilon_{\bar{\tau}_{i} \sigma_{i}}\left(U_{i}, U_{i}^{\dagger}\right)> \\
& =\int_{\Sigma\left(\mathcal{M} ;\left\{\sigma_{i}, \tau_{i}\right\}\right)} d \nu e^{-\frac{(n+\tilde{n}) \lambda A}{2}} \frac{(-1)^{i+\tilde{t}} N^{2-2 g-\beta}}{\left|S_{\nu}\right|}\left(\prod_{j=1}^{\max (0,-I)}(-1)^{x_{j}}\right)
\end{aligned}
$$

where the maps in $\Sigma\left(\mathcal{M} ;\left\{\sigma_{i}, \tau_{i}\right\}\right)$ are those covering maps with $2-2 G-b \Omega$-points satisfying the condition that the permutations on the orientation-preserving and orientation-reversing sheets of the cover of the boundary component $E_{i}$ are given by $\sigma_{i}, \tau_{i}$ respectively. From this result, it is clear that in the coupled theory, the generalized $\Upsilon$ functions are the natural basis for the Hilbert space in the string theory.

The next set of formulae we will need describe how partition functions on two manifolds with boundaries can be glued together to form a partition function on the manifold formed 


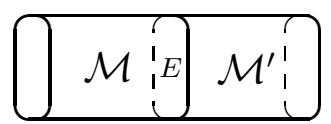

(a)

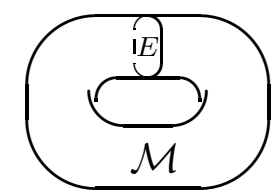

(c)

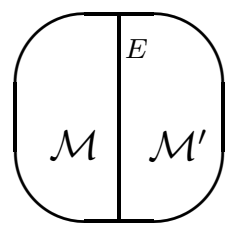

(b)

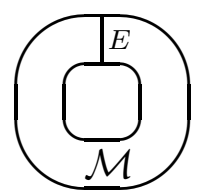

(d)

Figure 1: Gluing manifolds along a circle and a segment

by joining the two manifolds along a common edge. In general, if two manifolds $\mathcal{M}$ and $\mathcal{M}^{\prime}$ are joined along a common edge $E$, the partition function of the combined manifold

$$
\mathcal{N}=\mathcal{M} \bigcup_{E} \mathcal{M}^{\prime}
$$

is given by taking the product of the partition functions of $\mathcal{M}$ and $\mathcal{M}^{\prime}$ and integrating over the holonomy of the gauge field along the edge $E$. There are two cases of interest; the first is when the edge $E$ is a closed 1-manifold homeomorphic to $S^{1}$ (Figure 11a), the second case is when $E$ is a line segment with boundary (Figure [1]b).

It is also necessary to consider the case where a manifold $\mathcal{M}$ is glued to itself along an edge that appears twice (with opposite orientation) in the boundary of $\mathcal{M}$ (see Figure 1 $\mathrm{c}, \mathrm{d}$ ). In the basis of irreducible characters, the gluing formulae are straightforward. In the case of gluing along a circle, the relevant result is the orthonormality of the characters

$$
\int d U \chi_{R}(U) \chi_{R^{\prime}}\left(U^{\dagger}\right)=\delta_{R, R^{\prime}}
$$

In the case of gluing along a segment, the formula is

$$
\int d U \chi_{R}(V U) \chi_{R^{\prime}}\left(U^{\dagger} W\right)=\frac{\delta_{R, R^{\prime}}}{\operatorname{dim} R} \chi_{R}(V W)
$$

We wish to convert (4.11) and (4.12) into gluing formulae for partition functions expressed in terms of the $\Upsilon_{\sigma}$ functions. By using the Frobenius relations, the gluing formula on a circle 
follows immediately; we have

$$
\begin{aligned}
\int d U \Upsilon_{\sigma}(U) \Upsilon_{\tau}\left(U^{\dagger}\right) & =\int d U \sum_{R, R^{\prime}} \chi_{R}(\sigma) \chi_{R^{\prime}}(\tau) \chi_{R}(U) \chi_{R^{\prime}}\left(U^{\dagger}\right) \\
& =\sum_{R} \chi_{R}(\sigma) \chi_{R}(\tau)=\frac{\delta_{T_{\sigma}, T_{\tau}} n !}{\left|T_{\sigma}\right|}
\end{aligned}
$$

where $T_{\sigma}$ is the conjugacy class of elements in the symmetric group that contains $\sigma$. This formula can be interpreted in the following fashion: Assume we have two manifolds $\mathcal{M}$ and $\mathcal{M}^{\prime}$, each of which has a boundary component $E \approx S^{1}$. Because we are working in a single sector of the theory, the orientation of $E$ is taken to be opposite on the two manifolds. If the partition functions on $\mathcal{M}$ and $\mathcal{M}^{\prime}$ are given by sums over covers with $I$ and $I^{\prime} \Omega$-points respectively, then we can combine the partition functions in a term by term fashion through (4.13). Given two $n$-fold covers of $\mathcal{M}$ and $\mathcal{M}^{\prime}$ whose boundary components above $E$ are described by the conjugacy classes $T_{\sigma}$ and $T_{\tau}$, the covers can only be joined along $E$ when $T_{\sigma}=T_{\tau}$. When these conjugacy classes are equal, the number of ways that the covering spaces can be glued together is exactly the number $C_{\sigma}$ of elements of $S_{n}$ that commute with an element $\sigma \in T_{\sigma}$. This number is exactly $C_{\sigma}=n ! /\left|T_{\sigma}\right|$. Every cover of the joined manifold $\mathcal{N}$ can be formed in exactly such a fashion by gluing together covers of $\mathcal{M}$ and $\mathcal{M}^{\prime}$. Thus, (4.13) states that the partition function for $\mathcal{N}$ is given by taking the sum over covers of $\mathcal{N}$ that are formed by joining together covers of $\mathcal{M}$ and $\mathcal{M}^{\prime}$ in all possible ways along the common boundary. Note that in this gluing process, no new $\Omega$-points are introduced. The argument given here also shows that when a manifold $\mathcal{M}$ is glued to itself along a circle $E \approx S^{1}$, as in Figure $1 \mathrm{c}$, the partition function of the new manifold is given by gluing all covers of $\mathcal{M}$ together along $E$, without introducing new $\Omega$-points.

Generalizing the formula for gluing along a circle to the coupled theory is again a straightforward application of the generalized Frobenius relations. As shown in Appendix A, this gluing formula is given by

$$
\int d U \Upsilon_{\bar{\tau} \sigma}\left(U, U^{\dagger}\right) \Upsilon_{\bar{\tau}^{\prime} \sigma^{\prime}}\left(U, U^{\dagger}\right)=\delta_{T_{\sigma}, T_{\tau^{\prime}}} \delta_{T_{\sigma^{\prime}}, T_{\tau}} C_{\sigma} C_{\tau} .
$$

Again, this formula has the interpretation that when two manifolds are glued together along a circle (or a manifold is glued to itself), the partition function is joined in the string picture by gluing all covers together in all possible ways, without introducing new $\Omega$-points.

We observe in passing that the gluing formula (4.14) can be used to construct the partition function on a manifold of any genus by gluing together spheres with 1,2, and 3 boundary 


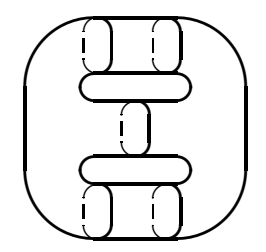

Figure 2: A genus 2 surface built from spheres with boundary

components. These three objects, along with the gluing formula (4.14), form a fundamental set of vertices that can be viewed as yielding a set of "Feynman rules" for the string theory. The sphere with three boundary components ("pair of pants") is like a vertex; this partition function is given by a sum over covering maps with one $\Omega^{-1}$-point. The sphere with two boundary components (the annulus or the cylinder) is like a propagator. This partition function is given by a sum over covering maps with no twist points of either type. The sphere with one boundary component (the disk or cap), is analogous to a tadpole and has a partition function given by a sum over covering maps with a single $\Omega$-point. Since gluing along a circle introduces no new twist points, it is possible to glue together these fundamental objects to form a genus $G$ Riemann surface, with a total of $2-2 G \Omega$-points, where $\Omega^{-1}$-points are counted as -1 . Note that the effect of an $\Omega^{-1}$-point is exactly to cancel a single $\Omega$-point. As an example, in Figure 2 a genus 2 surface is built out of 2 vertices and 2 propagators for a total of $-2 \Omega$-points. Note that the viewpoint expressed here differs from the related familiar description of conformal and topological theories, in that we are gluing together the target space and not the string world sheet.

Now let us consider the formula needed to glue $\Upsilon$ functions along a line segment. The most general expression which will be of use is a formula for the integral

$$
\int d U U_{j_{1}}^{i_{1}} \cdots U_{j_{n}}^{i_{n}} U_{l_{1}}^{\dagger k_{1}} \cdots U_{l_{n}}^{\dagger k_{n}}
$$

From the symmetry of this expression under permutations of the indices, it is clear that the integral is given by a sum over Wick contractions with various permutations $\rho$ and unknown coefficients $W_{\rho}$,

$$
\int d U U_{j_{1}}^{i_{1}} \cdots U_{j_{n}}^{i_{n}} U_{l_{1}}^{\dagger k_{1}} \cdots U_{l_{n}}^{\dagger k_{n}}=\sum_{\rho, \sigma} W_{\rho} \delta^{i_{1}}{ }_{l_{\sigma_{1}}} \delta^{k_{(\rho \sigma)_{1}}}{ }_{j_{1}} \cdots \delta_{l_{\sigma_{n}}}^{i_{n}} \delta_{j_{n}}^{k_{(\rho \sigma)_{n}}}
$$

where $\sigma_{i}$ is the image of $i$ under the permutation $\sigma$ of the integers $1, \ldots, n$. To determine 
the coefficients $W_{\rho}$, we consider the related integral

$$
\begin{aligned}
\int d U(\operatorname{Tr} V U)^{n}\left(\operatorname{Tr} U^{\dagger} W\right)^{n} & =\int d U \sum_{R} d_{R} \chi_{R}(V U) \sum_{R^{\prime}} d_{R^{\prime}} \chi_{R^{\prime}}\left(U^{\dagger} W\right) \\
=\sum_{R} \frac{d_{R}^{2}}{\operatorname{dim} R} \chi_{R}(V U) & =\sum_{R} \frac{d_{R}}{N^{n}} \sum_{\rho} \chi_{R}\left(\Omega_{n}^{-1} \rho\right) \Upsilon_{\rho}(V W) \\
=\frac{n !}{N^{n}} \sum_{\rho} \delta\left(\Omega_{n}^{-1} \rho\right) \Upsilon_{\rho}(V W) & =\frac{n !}{N^{n}} \sum_{\rho} w_{\rho} \Upsilon_{\rho}(V W),
\end{aligned}
$$

where we have used (2.22). Comparing (4.17) to (4.16), we see that

$$
\begin{gathered}
W_{\rho}=\frac{w_{\rho}}{N^{n}}, \\
\int d U U^{i_{1}}{ }_{j_{1}} \cdots U^{i_{n}}{ }_{j_{n}} U^{\dagger k_{l_{1}}} \cdots U^{\dagger k_{n}{ }_{l_{n}}}=\sum_{\rho, \sigma} \frac{w_{\rho}}{N^{n}} \delta^{i_{1}}{ }_{l_{\sigma_{1}}} \delta^{k_{(\rho \sigma)}{ }_{j_{1}} \cdots \delta^{i_{n}}{ }_{l_{\sigma_{n}}} \delta^{k(\rho \sigma)_{n}}{ }_{j_{n}} .}
\end{gathered}
$$

This equation can be interpreted in terms of gluing together covering maps in a simple fashion. If the only term in (4.19) were the leading term corresponding to $\rho$ being the identity permutation, then the integral would simply give the well-known Wick contraction of all edges, that corresponds to gluing the edges of the covering spaces together in all possible ways [4]. The existence of the extra factor of $\rho$, however, indicates that the edges on one side or the other must be allowed to twist in an arbitrary fashion. Geometrically, this twisting is exactly described by putting an extra $\Omega^{-1}$-point on one side of the edge before gluing, and then applying the simple Wick contraction prescription. Thus, we can describe the gluing together of the manifolds $\mathcal{M}$ and $\mathcal{M}^{\prime}$ along a common segment $E$ by inserting an extra $\Omega^{-1}$-point in $\mathcal{M}$, and then gluing together all covers of $\mathcal{M}$ (including the extra twist) with all covers of $\mathcal{M}^{\prime}$. Similarly, when a manifold $\mathcal{M}$ is glued to itself along a segment $E$, the partition function of the new manifold is formed by inserting an extra $\Omega^{-1}$-point in $\mathcal{M}$ before gluing the covering spaces together by a Wick contraction.

We have thus completed a description of all gluing processes in a single sector of the theory. When we consider the full theory, it is again possible to calculate the result of gluing together manifolds along an edge segment using (4.19); however, this process does not have such a simple description as in a single sector. The leading order terms, of course, combine according to the Wick contraction; however, the lower order terms combine in a more complicated fashion since the basis functions $\Upsilon_{\bar{\tau} \sigma}\left(U, U^{\dagger}\right)$ contain both factors of $U$ and $U^{\dagger}$. In order to achieve a simple geometric understanding of this formula in the coupled

\footnotetext{
${ }^{\dagger}$ A similar formula was derived by S. Samuel [7] .
} 
theory, one must either contract the indices of (4.19) with other edges around a boundary, or one must introduce a complicated set of orthogonal polynomials in $U$ and $U^{\dagger}$ with an arbitrary number of indices.

It is appropriate at this point to comment on past attempts to derive a string representation of QCD. This effort has a long history [8]. Most of the attempts were based on the strong coupling expansion of the Wilson lattice gauge theory, which to leading order can be written as a sum over surfaces with simple weights. One expanded the Wilson action,

$\exp \left[-1 / g^{2} \operatorname{Tr}\left(U_{P}+U_{P}^{\dagger}\right)\right]$, where $U_{P}$ is the holonomy around an elementary plaquette of the lattice, in powers of $1 / g^{2}$. Then one used the integral (4.19) to join the plaquettes to form a surface. The culmination of this enterprise was the work of Kazakov [9], of Kostov [10] [11] and of O'Brien and Zuber [12. They gave rules that expressed the strong coupling expansion as a complicated sum over surfaces with an infinite number of local factors. Since this approach was based on an expansion about infinite coupling the nature of the continuum limit was very unclear. In addition, the discovery of large $N$ phase transitions [13 raised additional suspicions about the relevance of these results to the continuum theory.

The approach that we are following is very different. As proposed in [3], we are using the exact solution of two dimensional QCD to construct an equivalent string theory, which we then hope to continue to higher dimensions. The exact lattice representation we employ is one for which the coupling is always in the weak coupling phase. The strong coupling limit of the Wilson action is not accessible using this formulation of the theory. In higher dimensions of course this method is not available. If we understood enough about QCD to derive its string theory representation then the string theory would be superfluous. The reason that we need such a representation is precisely because we do not have analytic control over the theory in the infra-red. Instead we hope to employ the lessons of two dimensions and wisdom of string theory to guess the QCD string theory in four dimensions.

\section{Wilson loops}

We now wish to calculate the vacuum expectation values (VEV's) of Wilson loops in $\mathrm{QCD}_{2}$ and to relate these VEV's to string theory maps. We derive in this section a general formula that can be used to compute vacuum expectation values of arbitrary products of Wilson loops on an arbitrary 2-manifold in a single sector of the theory. We will prove this formula by using the Wilson loops to cut the manifold into pieces, each of which is a manifold with 
boundary, and then gluing the pieces together along the loops using the gluing formulae from the previous section. We find that the vacuum expectation value of any product of Wilson loops is given by a sum similar to (2.28), where each of the regions bounded by the loops contains some number of $\Omega$-points or $\Omega^{-1}$-points, and where the covering space has a boundary that covers the Wilson loops in a fashion that is determined by the labels on the loops. We do not give a general formula here for the expectation values of Wilson loops in the coupled theory. We do, however, describe an algorithm by means of which an arbitrary such expectation value can be computed. Several simple examples of Wilson loop calculations in the coupled theory are described in section 7 . To derive a general formula for the full theory would involve a similar analysis to that performed in this section; however, the technical details become far more complex. Thus, for most of this section, we will confine our discussion to a single sector of the theory.

As mentioned in the Introduction, in the string theory the natural type of label to associate with a Wilson loop $\gamma$ is an integer $k$ and a permutation $\sigma \in S_{k}$. The VEV of the Wilson loop with this label is defined to be $\left\langle\Upsilon_{\sigma}(U)\right\rangle$, where $U$ is the holonomy of the gauge field around the Wilson loop. It should be clear from the discussion of manifolds with boundaries in the previous section that these are the most natural observables from the point of view of string maps, since the functions $\Upsilon_{\sigma}$ are the natural basis for the Hilbert space in the string theory picture. These observables are related to the more familiar vacuum expectation values of $\left\langle\chi_{R}(U)\right\rangle$ by the Frobenius relations. In a single sector of the theory, it is sufficient to consider VEV's of functions $\Upsilon_{\sigma}(U)$; in the coupled theory, one would like to compute VEV's of arbitrary functions $\Upsilon_{\bar{\tau} \sigma}\left(U, U^{\dagger}\right)$. We will denote VEV's in the orientation-preserving sector by $\left\langle\Upsilon_{\sigma}(U)\right\rangle_{+}$.

We assume that the manifold $\mathcal{M}$ is provided with a preferred orientation. If we consider a local region of the manifold to be embedded in Euclidian 3-space, we can relate the orientation of $\mathcal{M}$ to a normal vector field $\hat{n}(z)$. Each Wilson loop is also associated with some orientation, according to the direction in which the gauge field is integrated. Thus, it is possible to distinguish the two sides of a Wilson loop at a point. We will use the labels "inside" and "outside" to denote these two sides. If $\hat{\gamma}(z)$ is the oriented tangent vector to $\gamma$ at a point $z$, then the vector $\hat{n}(z) \times \hat{\gamma}(z)$ points into the region on the inside of $\gamma$ (see Figure (3). When we are working in a single sector of the gauge theory on a manifold with boundary, we are restricting to covering spaces that lie on a specific side of the boundary curves $E_{i}$. We will set the convention that in the orientation-preserving sector of the the- 


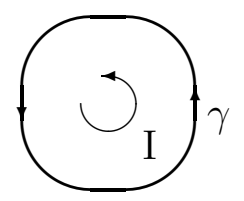

Figure 3: the inside (I) and outside $(\mathrm{O})$ of an oriented closed curve $\gamma$

ory, the covering spaces must lie on the "outside" of the boundary curves. Although this choice of notation may seem odd, it leads to a more intuitive description of Wilson loops; the orientation-preserving sheets of a cover that are bounded by a Wilson loop always lie on the inside of the loop.

Given an arbitrary set of Wilson loops $\gamma_{i}$ carrying the labels $\left(k_{i}, \sigma_{i} \in S_{k_{i}}\right)$, we are interested in computing the VEV

$$
\left\langle W_{\left\{\gamma_{i}, \sigma_{i}\right\}}\right\rangle_{+}=\left\langle\prod_{j} \Upsilon_{\sigma_{j}}\left(U_{j}\right)\right\rangle_{+},
$$

where $U_{j}$ is the holonomy around the $j$ th Wilson loop $\gamma_{j}$. We will assume that the Wilson loops do not have intersection points where more than two loops intersect (including selfintersections). Wilson loops that do not satisfy this criterion can be computed by taking the limit of a set of Wilson loops that do satisfy the criterion. With this assumption, we can consider the Wilson loops $\gamma_{j}$ to form a graph $\Gamma$ on $\mathcal{M}$, with vertices $V_{1}, \ldots, V_{v}$ at intersection points and edges $E_{1}, \ldots, E_{e}$ connecting the vertices along the Wilson loops (see Figure 4 ). By cutting along the Wilson loops, $\mathcal{M} \backslash\left(\bigcup \gamma_{i}\right)$ is divided into a disjoint union of connected regions $M_{j}$. If we denote the areas of these regions by $A_{j}$, then the $\mathrm{VEV}$ of $W_{\left\{\gamma_{i}, \sigma_{i}\right\}}$ is a function of the $A_{j}$.

We note in passing that the VEV of a Wilson loop with an arbitrary label $(k, \sigma)$ can be computed as the limit of the VEV of a Wilson loop in the fundamental representation $(k=1)$ as the areas of certain regions go to 0. For example, the VEV of the trivial Wilson loop on the sphere (Figure 11(a)) with the label $\left(2, \sigma_{2}=\left[2^{1}\right]\right)$ is equal to the VEV of the double Wilson loop on the sphere as the intermediate area goes to 0 (Figure 11(b) as $A_{3} \rightarrow 0$ ). Nonetheless, we will now give the formula for the expectation value of a product of loops with arbitrary labels.

The result is computed as follows: to each region $M_{j}$ we assign a number of $\Omega$-points, 


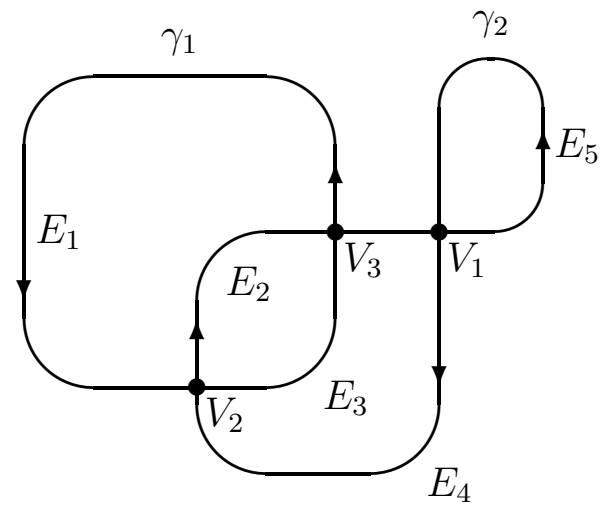

Figure 4: A graph $\Gamma$ formed by Wilson loops $\gamma_{1}, \gamma_{2}$ with edges $E_{a}$, vertices $V_{b}$

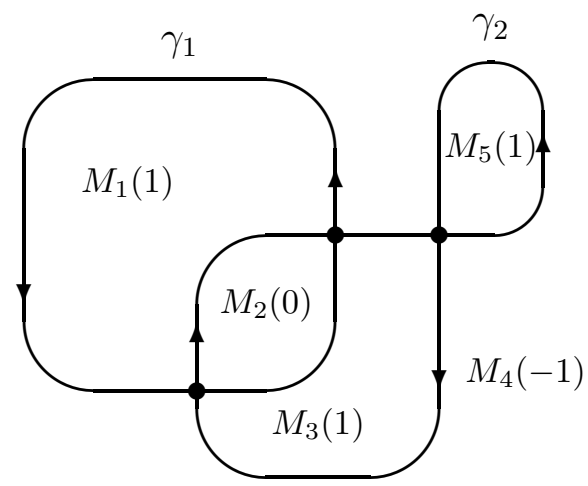

Figure 5: Numbers of $\Omega$-points in each region $M_{j}\left(I_{j}\right)$

$I_{j}$, according to the parts of the graph $\Gamma$ that lie on the boundary of $M_{j}$. Specifically, each region $M_{j}$ contributes $2-2 G_{j}-b_{j}$ to $I_{j}$, where $G_{j}$ is the genus of $M_{j}$ and $b_{j}$ is the number of boundary components. In addition, each edge of $\Gamma$ contributes an $\Omega^{-1}$-point to the region on its inside, and each vertex of $\Gamma$ contributes an $\Omega$-point to the region that lies on the inside of both edges that pass through that vertex. As an example, in Figure 5, the numbers of $\Omega$-points are shown for each region of the sphere after cutting along the Wilson loops from Figure 4 .

We define $\Sigma_{+}\left(\mathcal{M} ;\left\{\gamma_{i}, \sigma_{i}\right\}\right)$ to be the set of orientation-preserving covers of $\mathcal{M}$ with the usual singularities, $I_{j} \Omega$-points in the region $M_{j}$, and a boundary that covers the Wilson loops $\gamma_{i}$ with the permutations $\sigma_{i}$. The expectation value of the product of Wilson loops can 


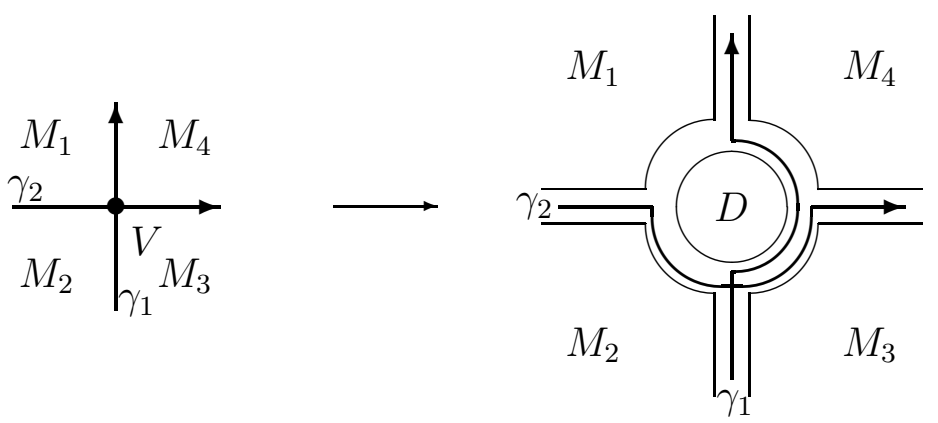

Figure 6: a disk $D$ at the intersection $V$ of two Wilson loops

be expressed as a sum over the covers in this set,

$$
\left\langle W_{\left\{\gamma_{i}, \sigma_{i}\right\}}\right\rangle_{+}=\int_{\Sigma_{+}\left(\mathcal{M} ;\left\{\gamma_{i}, \sigma_{i}\right\}\right)} d \nu e^{-\frac{n \lambda A}{2}} \frac{(-1)^{i} N^{2-2 g-\beta}}{\left|S_{\nu}\right|} \prod_{j} \prod_{k=1}^{\max \left(0,-I_{j}\right)}(-1)^{x_{(j, k)}}
$$

where everything is defined as in (4.7), and $x_{(j, k)}$ is the number of twists at the $k$ th $\Omega^{-1}$-point in the region $M_{j}$.

We will now prove this assertion, by cutting the manifold along the Wilson loops and gluing together using the formulae from the previous section. In order to make the calculation clearer, it will be useful to define a set of infinitesimal disks $D_{1}, \ldots, D_{v}$ around the vertices $V_{i}$. We will assume that the Wilson loops are deformed so that the disk $D_{i}$ lies on the inside of the Wilson loops passing through the vertex $V_{i}$ (see figure 6). The gluing process can then be performed in the following order: first the partition functions over the regions $M_{j}$ are glued along the edges $E_{1}, \ldots, E_{e}$ using (4.19), then the disks $D_{i}$ are glued into the resulting manifold using (4.13).

When we begin this gluing process, the partition function over each region $M_{j}$ is described by a sum over covers of $M_{j}$ with $2-2 G_{j}-b_{j} \Omega$-points, as was demonstrated in the previous section. Each time we glue together two regions along an edge $E$, we must apply the gluing formula (4.19). Since some Wilson loop $\gamma_{i}$ passes along $E$, we must include an extra $k_{i}$ copies of $U$ in the left-hand side of this gluing formula (we assume that the edge is oriented consistently with $\gamma_{i}$ ). Clearly, this integral will only be non-zero when the number of sheets of the cover on the inside of $E$ (corresponding to factors of $U^{\dagger}$ ) exceeds the number of sheets on the outside (corresponding to factors of $U$ ) by $k_{i}$. Note that if the same region lies on both sides of the edge $E$, this condition is never satisfied, and the Wilson loop VEV must be 0 . We described the result of (4.19) geometrically by observing that this gluing formula was equivalent to twisting the boundaries on one side of the edge by an $\Omega^{-1}$-point, then gluing 
in all possible ways according to the Wick contraction. We can use this same geometric interpretation here; we must only ensure that the $\Omega^{-1}$-point does not mix edges from the Wilson loop with edges on the boundary of the outside region. This is easily done by using the $\Omega^{-1}$-point to twist the edges on the inside region (the factors of $U^{\dagger}$ ). This is equivalent to placing an extra $\Omega^{-1}$-point in the inside region, and accounts for the edge contribution to $I_{j}$ given above.

After we have glued the regions along all edges $E_{i}$ of $\Gamma$, it remains to glue in the disks $D_{i}$. Each disk $D$ is associated with a single $\Omega$-point. Because we have deformed the Wilson loops such that $D$ is on the inside of all loops, the number of sheets in the cover of the boundary of $D$ (the number of factors of $U$ ) is constant around the boundary. Thus, we may apply (4.13), and we find that the disk $D$ is added with no extra twists; the single $\Omega$-point from $D$ can be associated with the region on the inside of both Wilson loops (in Figure 6, $M_{1}$ ).

Thus, we have proven (5.2). This gives the general formula for the vacuum expectation value of an arbitrary product of Wilson loops in a single sector of the theory. The natural next problem is to generalize this result to the full, coupled theory. Although the vacuum expectation value of a specific Wilson loop in the full theory can be calculated using the formulae (4.19) and (4.14), we do not yet have such a simple description of the geometry of this computation. The difficulties that arise in the coupled theory arise from two related phenomena. First, a Wilson loop in the coupled theory can either bound an orientationpreserving sheet on the inside, or an orientation-reversing sheet on the outside. Second, the extra terms in the functions $\Upsilon_{\bar{\tau} \sigma}\left(U, U^{\dagger}\right)$, that arise from the necessity to suppress folds that would arise from contracting factors of $U$ and $U^{\dagger}$ in the function $\Upsilon_{\sigma}(U) \Upsilon_{\tau}\left(U^{\dagger}\right)$, make computations significantly more difficult.

The first place in the analysis where the coupled theory is more complicated is when one glues regions together along the edges $E_{i}$ of $\Gamma$. If a Wilson loop bounds sheets on both sides, it is impossible to avoid having the $\Omega^{-1}$-point mix the Wilson loop with edges of the boundaries. This problem can be avoided by restricting attention to Wilson loops carrying the fundamental representation of $S U(N)(k=1)$. As noted above, the expectation values of all Wilson loops with arbitrary labels can be related to such fundamental loops, so this is not a significant restriction. With this constraint, each edge of the Wilson loop only bounds any given cover on a single side, so the $\Omega^{-1}$-points arising from edges can be distributed as in the single sector theory. Note, however, that now the quantities $I_{j}$ depend upon the distribution of sheets in the cover. 


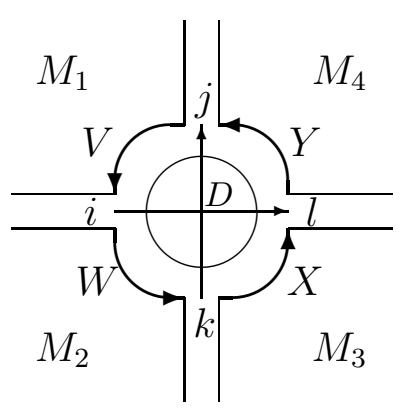

Figure 7: Intersection point in the coupled theory

Even though by this means it is possible to understand the gluing along edges simply, the final gluing of the disks raises more serious technical difficulties. Consider the disk $D$ depicted in Figure 0. We have broken up the boundary of $D$ into four segments with holonomies $V, W, X, Y$, corresponding to the segments that are contained in the boundaries of regions $M_{1}, M_{2}, M_{3}, M_{4}$. After gluing together these regions, the partition function will be a sum of terms whose dependence on $V, W, X, Y$ is described by a general polynomial $\psi_{j l}^{i k}(V, W, X, Y)$, whose transformation properties are indicated by the tensor indices. The remaining portions of the Wilson loops passing through this intersection are given by $\delta_{i}^{l}$ and $\delta^{j}{ }_{k}$ in the limit where the area of the disk $D$ goes to 0 . To calculate the effect of gluing in the disk, these Wilson loops must be contracted with the indices of $\psi$, and the holonomies $V, W, X, Y$ must be taken to 1 .

As an example of the type of situation that can arise in the coupled theory, consider the case where a cover of $\mathcal{M}$ in the neighborhood of $D$ has a single orientation-preserving cover in region $M_{1}$ and a single orientation-reversing cover in region $M_{3}$. In such a case, the $\Omega$-point usually associated with $D$ cannot be simply placed in one of the adjacent regions, since there is not a unique adjacent region containing all the local sheets of the cover. In this particular case, after the regions $M_{1}, M_{2}, M_{3}$, and $M_{4}$ have been glued together, the partition function will be proportional to $V_{j}^{i} X^{\dagger^{k}}{ }_{l}$. This result follows immediately from (4.19). To compute the contribution of the disk, we compute

$$
\lim _{V, X \rightarrow 1} \delta_{i}^{l} \delta_{k}^{j} V_{j}^{i} X^{\dagger^{k}}=N
$$

This result can be interpreted as indicating that the sheets of opposite orientation are connected at the vertex with a simple twist, with no additional tubes or extra structure allowed. We will compute several examples of Wilson loops with this type of vertex in Section 7.

We conclude this section with a brief description of how the contribution from a general 
vertex in the coupled theory can be computed; we do not have a simple geometric description of this computation, such as we have described in the single sector theory. We will use the notation $U=Y X W V$ for the total holonomy around the disk $D$, and the abbreviation $\Delta$ for the product of Wilson loop tensors $\delta_{i}^{l} \delta^{j}{ }_{k}$. We will drop the indices in most equations for clarity. The most general form for a function $\psi$ of the holonomies $V, W, X, Y$ around $D$ is given by the functions

$$
\begin{aligned}
& A_{\sigma, \tau}^{s, t}(V, W, X, Y)=\Upsilon_{\sigma}(U) \Upsilon_{\tau}\left(U^{\dagger}\right)\left(V U^{s}\right)_{j}^{i}\left(X^{\dagger} Y^{\dagger} U^{t} Y\right)_{l}^{k} \\
& B_{\sigma, \tau}^{s, t}(V, W, X, Y)=\Upsilon_{\sigma}(U) \Upsilon_{\tau}\left(U^{\dagger}\right)\left(V U^{s} Y\right)^{i}{ }_{l}\left(X^{\dagger} Y^{\dagger} U^{t}\right)^{k}{ }_{j}
\end{aligned}
$$

where $s, t$ range from $-\infty$ to $\infty$, and $\sigma, \tau$ are arbitrary permutations. If we compute the inner product on these functions by contracting indices and integrating, we find that

$$
\begin{aligned}
\int A_{\sigma, \tau}^{s, t} A_{\rho, v}^{\dagger r, u} & =\int d U \Upsilon_{\sigma+v}(U) \Upsilon_{\tau+\rho}\left(U^{\dagger}\right) \operatorname{Tr}\left(U^{s-r}\right) \operatorname{Tr}\left(U^{t-u}\right) \\
\int A_{\sigma, \tau}^{s, t} B_{\rho, v}^{\dagger r, u} & =\int d U \Upsilon_{\sigma+v}(U) \Upsilon_{\tau+\rho}\left(U^{\dagger}\right) \operatorname{Tr}\left(U^{s-r+t-u}\right) \\
\int B_{\sigma, \tau}^{s, t} B_{\rho, v}^{\dagger r, u} & =\int d U \Upsilon_{\sigma+v}(U) \Upsilon_{\tau+\rho}\left(U^{\dagger}\right) \operatorname{Tr}\left(U^{s-r}\right) \operatorname{Tr}\left(U^{t-u}\right) .
\end{aligned}
$$

These integrals can all be explicitly evaluated using (4.13).

There is a natural partial ordering defined on these functions. We define $(A, B)_{\sigma, \tau}^{s, t} \succ$ $(A, B)_{\sigma, \tau}^{s, t}$ when the number of factors of each term $V, W, X, Y, V^{\dagger}, W^{\dagger}, X^{\dagger}, Y^{\dagger}$ in the first function is greater than the number of corresponding terms in the second function. In this situation, we say that the first function "dominates" the second. Thus, for example,

$$
A_{(\cdot),(\cdot)}^{-1,1}=\left(W^{\dagger} X^{\dagger} Y^{\dagger}\right)^{i}{ }_{j}(W V Y)^{k}{ }_{l} \succ B_{(\cdot),(\cdot)}^{0,0}=(V Y)^{i}{ }_{l}\left(X^{\dagger} Y^{\dagger}\right)^{k}{ }_{j},
$$

where we denote by $(\cdot)$ the trivial permutation on 0 elements. On the other hand, considering the functions $A_{(\cdot),(\cdot)}^{0,0}=V_{j}^{i}\left(X^{\dagger}\right)^{k}{ }_{l}$ and $B_{(\cdot),(\cdot)}^{0,1}=(V Y)^{i}{ }_{l}(W V)^{k}{ }_{j}$, we find that neither function dominates the other.

In order to compute the effect of gluing in the disk $D$ when the covering space of $\mathcal{M} \backslash D$ has a fixed number of sheets in each region $M_{i}$, and a fixed permutation on the boundary of the cover around $D$, one must use the following general procedure. Associated with the boundary of the covering space, there is a particular function $\psi=A_{\sigma, \tau}^{s, t}$ or $\psi=B_{\sigma, \tau}^{s, t}$ defined by the permutation on the boundary of the cover. In the same way that the functions $\Upsilon_{\bar{\tau} \sigma}\left(U, U^{\dagger}\right)$, that provide a natural basis for the Hilbert space in the theory without Wilson 
loops, were defined by orthonormalizing the functions $\Upsilon_{\sigma}(U) \Upsilon_{\tau}\left(U^{\dagger}\right)$, we must orthonormalize the set of functions $A$ and $B$, to form a set of functions

$$
\begin{aligned}
\alpha_{\sigma, \tau}^{s, t} & =A_{\sigma, \tau}^{s, t}+\cdots \\
\beta_{\sigma, \tau}^{s, t} & =B_{\sigma, \tau}^{s, t}+\cdots,
\end{aligned}
$$

where the ellipses indicate subdominant terms, that are orthogonal to all subdominant terms; i.e., that satisfy

$$
\begin{array}{ll}
\int \alpha_{\sigma, \tau}^{s, t} \psi^{\dagger}=0, & \alpha_{\sigma, \tau}^{s, t} \succ \psi \\
\int \beta_{\sigma, \tau}^{s, t} \psi^{\dagger}=0, & \beta_{\sigma, \tau}^{s, t} \succ \psi .
\end{array}
$$

The function associated with the cover of $\mathcal{M} \backslash D$ will then be the function $\alpha$ or $\beta$ with a leading term consistent with the boundary of the cover. To compute the effect of gluing in the disk, we can then apply the formulae

$$
\begin{aligned}
\lim _{V, W, X, Y \rightarrow 1} \Delta \alpha_{\sigma, \tau}^{s, t} & =N^{1+K_{\sigma}+K_{\tau}}, \\
\lim _{V, W, X, Y \rightarrow 1} \Delta \beta_{\sigma, \tau}^{s, t} & =N^{2+K_{\sigma}+K_{\tau}} .
\end{aligned}
$$

We illustrate this general formalism with several examples. First, consider the covering described above, where $M_{1}$ has a single orientation-preserving covering sheet and $M_{3}$ has an orientation-reversing sheet. The function describing this cover is

$$
\alpha_{(\cdot),(\cdot)}^{0,0}=A_{(\cdot),(\cdot)}^{0,0}=V_{j}^{i} X^{\dagger}{ }_{l}^{k} .
$$

Computing the effect of gluing in the disk, we have

$$
\lim _{V, W, X, Y \rightarrow 1} \Delta \alpha_{(\cdot),(\cdot)}^{0,0}=N
$$

As mentioned above, this has the effect of gluing together the sheets of the cover with a simple twist and no tubes.

For another example, consider the situation where an orientation-preserving sheet covers regions $M_{1}, M_{2}, M_{4}$ and an orientation-reversing sheet covers regions $M_{2}, M_{3}, M_{4}$. The function associated with the boundary permutation is $\left(W^{\dagger} X^{\dagger} Y^{\dagger}\right)_{j}^{i}(W V Y)^{k}{ }_{l}=A_{(\cdot),(\cdot)}^{-1,1}$. Thus, we must find the function $\alpha_{(\cdot),(\cdot)}^{-1,1}$. This function can be computed by writing a sum of all subdominant terms with arbitrary coefficients

$$
\alpha_{(\cdot),(\cdot)}^{-1,1}=A_{(\cdot),(\cdot)}^{-1,1}+a A_{(\cdot),(\cdot)}^{0,0}+b B_{(\cdot),(\cdot)}^{0,0}+c B_{(\cdot),(\cdot)}^{-1,1}
$$


Taking the inner product with the subdominant terms, we have

$$
\begin{gathered}
\int \alpha_{(\cdot),(\cdot)}^{-1,1}\left(A_{(\cdot),(\cdot)}^{0,0}\right)^{\dagger}=1+a N^{2}+b N+c N=0, \\
\int \alpha_{(\cdot),(\cdot)}^{-1,1}\left(B_{(\cdot),(\cdot)}^{0,0}\right)^{\dagger}=N+a N+b N^{2}+c=0, \\
\int \alpha_{(\cdot),(\cdot)}^{-1,1}\left(B_{(\cdot),(\cdot)}^{-1,1}\right)^{\dagger}=N+a N+b+c N^{2}=0 .
\end{gathered}
$$

Thus, $a=1 / N^{2}, b=c=-1 / N$, so

$$
\alpha_{(\cdot),(\cdot)}^{-1,1}=A_{(\cdot),(\cdot)}^{-1,1}+\frac{1}{N^{2}} A_{(\cdot),(\cdot)}^{0,0}-\frac{1}{N} B_{(\cdot),(\cdot)}^{0,0}-\frac{1}{N} B_{(\cdot),(\cdot)}^{-1,1}
$$

Taking the limit and contracting with the remaining Wilson loops,

$$
\Delta \alpha_{(\cdot),(\cdot)}^{-1,1}=-N\left(1-\frac{1}{N^{2}}\right)
$$

Thus, the effect of such a vertex is to join the sheets with a twist, allowing an orientationreversing tube between the sheets, and to introduce an extra factor of -1 . We will consider an example of a Wilson loop with such a vertex in Section 7; we show there that this result is in agreement with an earlier calculation by Kazakov using loop equations 16.

\section{Examples of partition functions}

We will now give a variety of examples of calculations in the string theory picture. We will primarily perform explicit calculations for the terms corresponding to covering maps with small winding number, corresponding to representations $\bar{S} R$ where $S$ and $R$ are described by Young tableaux with a small number of boxes. In this section and the next, we will order the terms according to the number of sheets in the covering spaces, rather than according to powers of $1 / N$. For $G>1$, for each power of $1 / N$ it is only necessary to compute the terms corresponding to covering spaces with a finite number of sheets, since $g \geq 1+n(G-1)$.

We recall from [4] that for a manifold $\mathcal{M}$ of genus $G$ and area $A$, the free energy

$$
\mathcal{W}(G, \lambda A, N)=\ln \mathcal{Z}(G, \lambda A, N)
$$

describes a string theory identical to that described by $\mathcal{Z}(G, \lambda A, N)$, except that the covering spaces (the string world-sheets) are restricted to be connected spaces. It is this connected string theory that we will use to calculate the sum over covering maps in the first example 
below. We can expand the partition function and the free energy explicitly in terms of coefficients $\eta_{g, G}^{n, i}$ and $\omega_{g, G}^{n, i}$

$$
\begin{aligned}
\mathcal{Z}(G, \lambda A, N) & =\sum_{g=-\infty}^{\infty} \sum_{n} \sum_{i} \eta_{g, G}^{n, i} e^{-\frac{n \lambda A}{2}}(\lambda A)^{i} N^{2-2 g} . \\
\mathcal{W}(G, \lambda A, N) & =\sum_{g=-\infty}^{\infty} \sum_{n} \sum_{i} \omega_{g, G}^{n, i} e^{-\frac{n \lambda A}{2}}(\lambda A)^{i} N^{2-2 g} .
\end{aligned}
$$

We can perform a similar expansion for the partition function and free energy in a single sector,

$$
\begin{aligned}
Z^{+}(G, \lambda A, N) & =\sum_{g=-\infty}^{\infty} \sum_{n} \sum_{i} \zeta_{g, G}^{n, i} e^{-\frac{n \lambda A}{2}}(\lambda A)^{i} N^{2-2 g} . \\
W^{+}(G, \lambda A, N) & =\sum_{g=-\infty}^{\infty} \sum_{n} \sum_{i} \xi_{g, G}^{n, i} e^{-\frac{n \lambda A}{2}}(\lambda A)^{i} N^{2-2 g} .
\end{aligned}
$$

The coefficients $\xi_{g, G}^{n, i}$ when $2(g-1)=2 n(G-1)+i$ correspond to the $n$-fold orientationpreserving covering maps from a connected genus $g$ Riemann surface to $\mathcal{M}$ with $i$ branch points. When $i=0$, these terms count the number of connected unbranched $n$-fold covers of $\mathcal{M}$, where each cover counts as $1 /\left|S_{\nu}\right|$. The coefficients $\zeta_{g, G}^{n, i}$ similarly correspond to the number of orientation-preserving covering maps from a possibly disconnected Riemann service to $\mathcal{M}$.

- $G=0$

As a first example, we consider the partition function of the connected theory (6.1) on the manifold $S^{2}$. In many ways this is the most complicated case since here there are contributions of maps of arbitrarily large winding number even for a fixed power of $1 / N$.

In a single sector, the free energy is given by

$$
W^{+}(0, \lambda A, N)=\int_{\tilde{\Sigma}_{+}\left(S^{2}\right)} d \nu e^{-\frac{n \lambda A}{2}} \frac{(-1)^{i} N^{2-2 g}}{\left|S_{\nu}\right|}
$$

where the covering maps in $\tilde{\Sigma}_{+}\left(S^{2}\right)$ are continuous maps from connected spaces to $S^{2}$ that have exactly two $\Omega$-points. We will now calculate the contributions to (6.6) for $n=1$ and $n=2$.

When $n=1$, clearly there is only one covering space; this cover has a single sheet and no singularities. When there is only a single sheet, there can be no branch points and no tubes, and the effect of $\Omega$-points is restricted to the trivial permutation, so the contribution 
to $W^{+}(0, \lambda A, N)$ from maps with $n=1$ is given by the trivial single sheeted cover with all possible contracted handles. We recover the full contribution by summing over all possible locations of handles;

$$
W_{1}^{+}(0, \lambda A, N)=N D_{1}(\lambda A, N)=e^{-\frac{\lambda A}{2}} \sum_{h} \frac{(\lambda A)^{h}}{2^{h} h !} N^{2(1-h)}=N^{2} e^{-\frac{\lambda A}{2}+\frac{\lambda A}{2 N^{2}}},
$$

where by $D_{1}(A)$ we denote the $n=1$ contribution to the partition function on a disc of area $A$. We can easily verify this expression in $\mathrm{QCD}_{2}$; the exponent is just $-(\lambda / 2 N) C_{2}(\square)$, where the Casimir of the fundamental representation $(\square)$ is $N-1 / N$.

When $n=2$, the cover has two sheets. The only possible non-trivial permutation on these sheets is the permutation that switches sheets $(P)$; this is the permutation that every branch point must give, and is the only non-trivial permutation that may be produced by the $\Omega$-points. Thus $\Omega_{2}=1+P / N$, and $\Omega_{2}^{2}=\left(1+1 / N^{2}\right)+2 P / N$. The total number of permutations $P$ produced by branch points or $\Omega$-points must be even, since the covering space has no boundary. In addition the sheets may be connected by orientation preserving tubes. If there are no branch points and the permutation from each $\Omega$-point is trivial, then there must be at least one tube, or the covering space would be disconnected. Every covering space that has no contracted handles has a symmetry factor of $1 / 2$, since the two sheets are identical. Thus, the contribution to the free energy from maps with $n=2$ is given by,

$$
\begin{aligned}
W_{2}^{+}(0, \lambda A, N) & =e^{-\lambda A}\left[\frac{1}{2} \sum_{h} \frac{(\lambda A)^{h}}{h !} N^{-2 h}\right] \\
& \cdot\left[\sum_{t} \frac{(\lambda A)^{t}}{t !} N^{-2 t} \sum_{i} \frac{(\lambda A)^{2 i}}{(2 i) !} N^{2(2-i)}\left(1+\frac{1}{N^{2}}+\left(\frac{2}{N}\right)\left(\frac{-\lambda A}{(2 i+1) N}\right)\right)-N^{4}\right] \\
& =e^{-\lambda A}\left[N^{2}\left(\frac{1}{2}-\frac{1}{2} \lambda A+\frac{1}{4}(\lambda A)^{2}\right)+\mathcal{O}(1)\right]
\end{aligned}
$$

The subtraction of the $N^{4}$ term is to remove the case of no branchpoints, no tubes and trivial $\Omega$-points, that is disconnected. It is easily seen that this agrees with $\mathrm{QCD}_{2}$ by rewriting the result as

$$
\begin{aligned}
& W_{2}^{+}(0, \lambda A, N)= \\
& \quad\left(\frac{N(N+1)}{2}\right)^{2} e^{-\frac{\lambda A}{2 N}\left(2 N+2-\frac{4}{N}\right)}+\left(\frac{N(N-1)}{2}\right)^{2} e^{-\frac{\lambda A}{2 N}\left(2 N-2-\frac{4}{N}\right)}-\frac{N^{4}}{2} e^{-\frac{\lambda A}{N}\left(N-\frac{1}{N}\right)},
\end{aligned}
$$

and recognizing the first term as the contribution of the representation $\square$ to the partition 
function on the sphere, the second as the contribution of $\boxminus$, and the third as the subtraction of the disconnected parts.

We can combine these results to calculate the contributions to the coupled free energy $\mathcal{W}(0, \lambda A, N)$ from covers with $n \leq 2$. The coupled free energy is given by

$$
\begin{aligned}
& \mathcal{W}(0, \lambda A, N)= \\
& \quad 2\left(W_{1}^{+}(0, \lambda A, N)+W_{2}^{+}(0, \lambda A, N)\right) \\
& \quad+W_{1}^{+}(0, \lambda A, N)^{2}\left[\sum_{\tilde{t}} \frac{(-\lambda A)^{\tilde{t}}}{\tilde{t} !} N^{-2 \tilde{t}}\left(1-\frac{1}{N^{2}}\right)^{2}-1\right]+\mathcal{O}\left(e^{-\frac{3 \lambda A}{2}}\right) .
\end{aligned}
$$

The term quadratic in $W_{1}^{+}$arises from a 2-fold cover of $S^{2}$ consisting of a single-sheeted cover of each orientation connected by orientation-reversing tubes. The term that is subtracted is the disconnected cover of this type without tubes.

This procedure can be continued to higher winding number maps. A very interesting calculation would be to calculate the exact leading order contribution (order $\left.N^{2}\right)$ to $\mathcal{W}(0, \lambda A, N)$ for arbitrary winding number. Using the methods of [3] this calculation can be treated by discrete orthogonal polynomials; however, so far this has not yielded a closed form solution. - $g=G=1$

The next example is that of maps from the torus onto the torus. For such maps there can be no branch points, tubes, contracted handles, or nontrivial twists. Thus, we must simply count the total number of topologically distinct covering of a torus by another torus. If we consider connected maps then we can only have maps of a given orientation, since orientation reversing tubes would increase $g$. Thus $\mathcal{W}(1, \lambda A)=2 W^{+}(1, \lambda A)+\mathcal{O}\left(N^{-2}\right)$.

Consider an $n$-sheeted cover of the torus. The two cycles of the world sheet torus must wind an integral number of times around the two cycles of the target space torus; $k$ times around one cycle and $q$ times around the other. Clearly $n=k q$. To determine how many topologically inequivalent maps there are with such windings one can draw a fundamental region of the world sheet torus on the lattice associated with the universal covering space of the target space torus. In Figure 8 an example is given of a six sheeted cover with windings 3 and 2. There are many such coverings; for a given value of $k$ they can be labeled by an integer $l$, where the world-sheet torus is defined by the vertices $(0,0),(k, 0),(l, q),(k+l, q)$ on the target space lattice. But the mapping class transformations of the world sheet can 


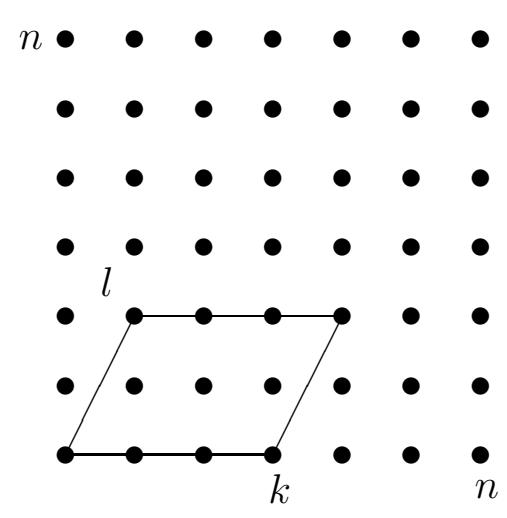

Figure 8: A 6-fold map of a torus with windings 3 and 2

transform $l \rightarrow l+m k$. Therefore the total number of inequivalent tori is

$$
C_{n}=\sum_{k \mid n} \sum_{l=0}^{k-1} 1=\sum_{k \mid n} k=\sum_{q \mid n} \frac{n}{q}
$$

where $k \mid n \Leftrightarrow k$ divides $n$. The symmetry factor associated with an $n$-fold cover of this type is always $n$, so the sum of the symmetry factor over all covers is given by

$$
\xi_{1,1}^{n, 0}=\sum_{q \mid n} \frac{1}{q}
$$

We can now calculate the leading term in $W^{+}(1, \lambda A)$ as

$$
\lim _{N \rightarrow \infty} W^{+}(1, \lambda A)=\sum_{n=1}^{\infty} \sum_{q \mid n} \frac{1}{q} x^{n}=\sum_{m=1}^{\infty} \sum_{q=1}^{\infty} \frac{1}{q} x^{m q}=-\sum_{m=1}^{\infty} \ln \left(1-x^{m}\right),
$$

where $x=\exp [-\lambda A / 2]$. Exponentiating the sum of connected maps then gives us the sum of all maps including disconnected ones. This should give the leading contribution to the QCD partition function on a torus as $N \rightarrow \infty$.

$$
\lim _{N \rightarrow \infty} Z^{+}(1, \lambda A)=\prod_{m} \frac{1}{\left(1-x^{m}\right)}=\eta(x)=\sum_{n} p(n) x^{n},
$$

where $p(n)$ is the number of partitions of $n$. This is easily seen to be identical to the QCD calculation,

$$
Z^{+}(1, \lambda A)=\sum_{n} \sum_{R \in Y_{n}} e^{-\frac{\lambda A C_{2}(R)}{2 N}}=\sum_{n_{1} \geq n_{2} \geq \cdots} x^{n_{1}+n_{2}+\cdots}\left(1+\mathcal{O}\left(\frac{1}{N}\right)\right)
$$




$$
=\sum_{n} p(n) x^{n}\left(1+\mathcal{O}\left(\frac{1}{N}\right)\right)
$$

This calculation complements the discussion in [4], where we calculated, using arguments along the lines of Section 2, the number of inequivalent disconnected maps of a torus onto a torus with winding number $n$ (namely $p(n)$.)

- $g=1+n(G-1)$

For our next example, we will compute the coefficients $\zeta_{g, G}^{n, 0}$ for arbitrary $G$ when $g=$ $1+n(G-1)$. These coefficients correspond to a sum over all disconnected orientationpreserving coverings of the genus $G$ manifold $\mathcal{M}$ without branch points, tubes, contracted handles, or nontrivial twists.

We will compute these coefficients by using the Feynman rules described in section 4 to construct the manifold $\mathcal{M}$ out of spheres with 1, 2 and 3 boundary components ("caps", "propagators" and "vertices"). Clearly when $n=1, \zeta_{g, G}^{n, 0}=1$, since there is a single cover of any genus Riemann surface with 1 sheet and no singular points. We will consider in particular the case $n=2$ while presenting an argument that easily generalizes to arbitrary $n$. In order to calculate the sum over double covering spaces of an arbitrary genus surface from the Feynman diagram point of view, we will need to know the set of nonsingular double covers of a disk, an annulus, and a sphere with 3 boundary components. As in the algebraic argument in section 2 , in order to count all covers with a factor of $1 /\left|S_{\nu}\right|$, it is simplest to label the sheets of the cover and to sum a factor of $1 / n$ ! over all distinct labelings. The boundaries of a 2 -fold cover are associated with elements of the symmetric group $S_{2}$. We denote these elements by $\sigma_{1}, \sigma_{2}$, where $\sigma_{1}$ is the identity element, corresponding to a disconnected 2-fold cover of the boundary component, and where $\sigma_{2}$ is the pairwise exchange corresponding to a connected 2-fold cover (see Figure 9).

In the Feynman diagram picture, we will associate with a disk of area $A$ a function $D(\sigma, A)$ on $S_{n}$, giving the sum of $N^{n} / n$ ! over all labeled covers of the disk with a boundary described by the permutation $\sigma$. Similarly, an annulus of area $A$ is described by a function $P(\sigma, \tau, A)$ where $\sigma$ and $\tau$ are the permutations describing the boundary components of covering spaces of the annulus, and a vertex of area $A$ is described by a function $V(\sigma, \tau, \rho, A)$. In general these functions contain covering maps with arbitrary singularities, and are rather complicated. Here, we have restricted to the simpler case where there are no singularities, so we can write fairly simple expressions for the functions. In fact, since the only covers of the sphere without singularities are given by multiple copies of the trivial 1 -fold cover, it is 


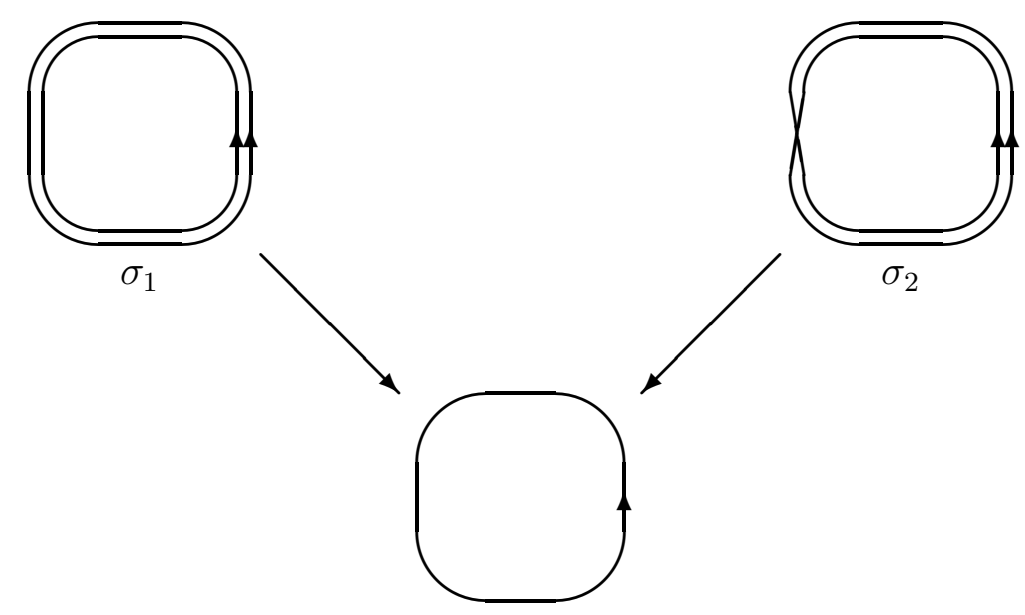

Figure 9: Elements of $S_{2}$ describing covers of a boundary component

easy to see that in our case,

$$
\begin{aligned}
D(\sigma, A) & =\frac{N^{n}}{n !} \delta(\sigma) \\
P(\sigma, \tau, A) & =\frac{1}{n !} \delta(\sigma \tau) \\
V(\sigma, \tau, \rho, A) & =\frac{1}{n ! N^{n}} \delta(\sigma \tau \rho) .
\end{aligned}
$$

When we glue together these fundamental objects along boundary loops, we must apply equation (4.13). In terms of the permutations and labels on the boundaries, this means that when we glue together an object with boundary $\sigma$ with another object with boundary $\tau$, we must sum over all possible permutations $\rho$ describing which labels are identified, and we must insert a delta function to enforce the condition that under this relabeling the permutation is preserved; i.e., that $\rho \sigma \rho^{-1}=\tau^{-1}$. (Note that we take the inverse of the permutation $\tau$, since the boundary associated with it is of the opposite orientation from the boundary associated with $\sigma$.) Thus, for instance, we can glue together a disk and a vertex along a common boundary to reproduce the function $P$ on an annulus,

$$
\sum_{\varrho, \rho, \varsigma} V(\sigma, \tau, \rho, A) D(\varsigma, B) \delta\left(\varrho \varsigma \varrho^{-1} \rho\right)=P(\sigma, \tau, A+B) .
$$

Similarly we can glue a disc onto the annulus to reproduce the disc,

$$
\sum_{\varrho, \tau, \varsigma} P(\sigma, \tau, A) D(\varsigma, B) \delta\left(\varrho \varsigma \varrho^{-1} \tau\right)=D(\sigma, A+B),
$$




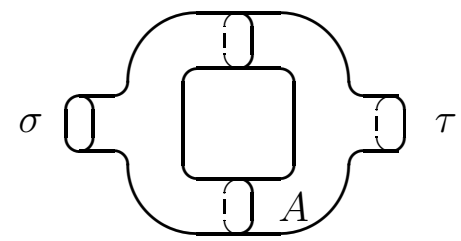

Figure 10: $T(\sigma, \tau, A)$ describes a torus with two boundary components

It is trivial to check that (6.21) and (6.22) are satisfied using (6.18), (6.19) and (6.20). Since in the restricted case we are interested in here, none of the elementary functions are dependent on $A$, we will henceforth drop the $A$ dependence of these functions. In a more general treatment, it would be necessary to include this dependence in all of the following equations.

As an intermediate step towards the result on a surface of arbitrary genus, we will find it useful to construct the function $T(\sigma, \tau, A)$ describing a torus with two boundaries. The function $T$ can be constructed by gluing together two vertices along two boundary loops, and is given by

$$
\begin{aligned}
T(\sigma, \tau) & =\sum_{\alpha, \beta, \rho, v, \rho^{\prime}, v^{\prime}} V(\sigma, \rho, v) V\left(\tau, \rho^{\prime}, v^{\prime}\right) \delta\left(\alpha \rho \alpha^{-1} \rho^{\prime}\right) \delta\left(\beta v \beta^{-1} v^{\prime}\right) \\
& =\frac{N^{-2 n}}{n !^{2}} \sum_{\alpha, \beta, \rho} \delta\left(\beta \rho^{-1} \sigma^{-1} \beta^{-1} \alpha \rho \alpha^{-1} \tau\right)
\end{aligned}
$$

The sum over nonsingular covering spaces of a surface of arbitrary genus $G$ can now be formed by gluing together $G$ copies of (6.23), to which we attach caps at the two ends. In general, gluing in a disk to a boundary component $\sigma$ has the effect of multiplying the function by $N^{n} \delta(\sigma)$, just as in (6.21) and (6.22), since the factor of $1 / n$ ! is canceled by the $n$ ! possible ways of gluing the labels together. Thus

$$
\begin{aligned}
\zeta_{g, G}^{n, 0} & =N^{n(2 G)} \sum_{\alpha_{1}, \ldots, \alpha_{G-1}} T\left(\sigma_{1}, \alpha_{1}\right) n ! T\left(\alpha_{1}, \alpha_{2}\right) n ! \ldots n ! T\left(\alpha_{G-1}, \sigma_{1}\right) \\
& =\frac{1}{n !} \sum_{\alpha_{1}, \rho_{1}, \ldots, \alpha_{G-1}, \rho_{G-1}} \delta\left(\prod_{i=1}^{G-1} \alpha_{i} \rho_{i} \alpha_{i}^{-1} \rho_{i}^{-1}\right)
\end{aligned}
$$

where in (6.24) we have used the gluing formula (4.13) and the fact that $T(\sigma, \tau)$ is a class function on $\sigma$ and $\tau$. This result agrees with (2.24). 
We will now perform this calculation explicitly for the case $n=2$. In this case, the functions $D$ and $V$ are given by

$$
\begin{gathered}
D\left(\sigma_{1}\right)=\frac{N^{2}}{2} ; \quad D\left(\sigma_{2}\right)=0 \\
V\left(\sigma_{1}, \sigma_{1}, \sigma_{1}\right)=V\left(\sigma_{1}, \sigma_{2}, \sigma_{2}\right)=\frac{1}{2 N^{2}} ; \quad V\left(\sigma_{1}, \sigma_{1}, \sigma_{2}\right)=V\left(\sigma_{2}, \sigma_{2}, \sigma_{2}\right)=0,
\end{gathered}
$$

where $V$ is symmetric in its 3 arguments. The function $T$ describing a torus with two boundary components is then given by

$$
T\left(\sigma_{1}, \sigma_{1}\right)=T\left(\sigma_{2}, \sigma_{2}\right)=\frac{2}{N^{4}} ; \quad T\left(\sigma_{1}, \sigma_{2}\right)=T\left(\sigma_{2}, \sigma_{1}\right)=0 .
$$

This function can be viewed as a matrix, $\langle\sigma|\mathbf{T}| \tau\rangle=N^{2 n} T(\sigma, \tau) \sqrt{\left|T_{\sigma}\right|\left|T_{\tau}\right|}$, in the space of conjugacy classes in $S_{2}$. The coefficient $\zeta_{2 G-1, G}^{2,0}$ is then given by

$$
\zeta_{2 G-1, G}^{2,0}=<\sigma_{1}\left|\mathbf{T}^{G}\right| \sigma_{1}>2 !^{G-1}=2^{2 G-1} .
$$

Similarly, for arbitrary $n$, we can compute the matrix $\left\langle\sigma\left|\mathbf{T}^{G}\right| \tau>\right.$ as a matrix in the space of conjugacy classes in $S_{n}$. We then have, $\zeta_{n G-n+1, G}^{n, 0}=<\sigma_{1}\left|\mathbf{T}^{G}\right| \sigma_{1}>n !{ }^{G-1}$, and we can compute the coefficient $\zeta_{n G-n+1, G}^{n, 0}$ by decomposing the identity permutation, $\mid \sigma_{1}>$, into a linear combination of eigenvectors of $\mathbf{T}$. For $n=3,4$, we get

$$
\begin{aligned}
& \zeta_{3 G-2, G}^{3,0}=2\left(6^{2 G-2}\right)+3^{2 G-2}, \\
& \zeta_{4 G-3, G}^{4,0}=2\left(24^{2 G-2}\right)+2\left(8^{2 G-2}\right)+12^{2 G-2} .
\end{aligned}
$$

For general $n$ we note that the normalized eigenvectors of $\mathbf{T}$ are simply given by the class functions $\chi_{R}(\tau) \sqrt{\left|T_{\tau}\right|} / \sqrt{n !}$, with eigenvalues given by $\frac{n !}{d_{R}^{2}}$. This is because

$$
\begin{aligned}
& N^{2 n} \sum_{T_{\tau}} \sqrt{\left|T_{\sigma}\right|}\left|T_{\tau}\right| T(\sigma, \tau) \chi_{R}(\tau) \\
& =\sum_{T_{\tau}} \frac{\sqrt{\left|T_{\sigma}\right|}\left|T_{\tau}\right|}{n !^{2}} \sum_{\alpha, \beta, \rho} \delta\left(\beta \rho^{-1} \sigma^{-1} \beta^{-1} \alpha \rho \alpha^{-1} \tau\right) \chi_{R}(\tau) \\
& =\sum_{\alpha, \beta, \rho} \sum_{T_{\tau}} \frac{\sqrt{\left|T_{\sigma}\right|}\left|T_{\tau}\right|}{n !^{3}} \sum_{R^{\prime}} \chi_{R^{\prime}}\left(\beta \rho^{-1} \sigma^{-1} \beta^{-1} \alpha \rho \alpha^{-1}\right) \chi_{R^{\prime}}(\tau) \chi_{R}(\tau) \\
& =\frac{\sqrt{\left|T_{\sigma}\right|}}{n !^{2}} \sum_{\alpha, \beta, \rho} \chi_{R}\left(\beta \rho^{-1} \sigma^{-1} \beta^{-1} \alpha \rho \alpha^{-1}\right)=\frac{\sqrt{\left|T_{\sigma}\right|}}{n !} \sum_{\alpha, \rho} \chi_{R}\left(\sigma^{-1} \alpha \rho \alpha^{-1} \rho^{-1}\right) \\
& =\frac{\sqrt{\left|T_{\sigma}\right|}}{d_{R}} \sum_{\rho} \chi_{R}\left(\alpha \rho \alpha^{-1} \rho^{-1}\right) \chi_{R}\left(\sigma^{-1}\right)=\frac{n ! \sqrt{\left|T_{\sigma}\right|}}{d_{R}^{2}} \chi_{R}\left(\sigma^{-1}\right)=\frac{n !}{d_{R}^{2}} \sqrt{\left|T_{\sigma}\right|} \chi_{R}(\sigma) .
\end{aligned}
$$


The identity has the decomposition, $\delta(\sigma)=\delta(\sigma) \sqrt{\left|T_{\sigma}\right|}=\sum_{R} \sqrt{\left|T_{\sigma}\right|} \chi_{R}(\sigma) \frac{d_{R}}{n !}$. Therefore,

$$
\zeta_{n G-n+1, G}^{n, 0}=\sum_{R}\left(\frac{n !}{d_{R}^{2}}\right)^{G}(n !)^{G-1}\left(\frac{d_{R}}{\sqrt{n !}}\right)^{2}=\sum_{R}\left(\frac{n !}{d_{R}}\right)^{2 G-2} .
$$

These results are equivalent to those given earlier from the algebraic point of view; however, this method of calculation gives a better picture of how these covering maps are described geometrically. We see that in the case of the torus, $G=1$, we recover again the number of possibly disconnected maps of the torus onto the torus with winding number $n$, namely $\zeta_{1,1}^{n, 0}=\sum_{R} 1=p(n)$.

We can easily check this result in $\mathrm{QCD}_{2}$, since for these maps we need only the leading term in the $1 / N$ expansion of the dimension and the quadratic Casimir, and thus

$$
Z^{+} \sim \sum_{R}\left(\frac{d_{R} N^{n}}{n !}\right)^{2-2 G} e^{-\frac{n \lambda A}{2}}
$$

in complete accord with (6.37). Also, the fact that the characters are the eigenvalues of $\mathbf{T}$ is clear from the QCD calculation, (4.4), that $T(\sigma, \tau)=\sum_{R} \frac{1}{d_{R}^{2} N^{2 n}} \chi_{R}(\sigma) \chi_{R}(\tau)$.

The terms we have computed here correspond to possibly disconnected orientationpreserving covers without singular points. The corresponding terms in the coupled theory are given by

$$
\eta_{n G-n+1, G}^{n, 0}=\sum_{m=0}^{n} \zeta_{m G-m+1, G}^{m, 0} \zeta_{(n-m) G-n+m+1, G}^{n-m, 0},
$$

since each nonsingular covering space can be divided into an orientation-preserving nonsingular covering space and a nonsingular orientation-reversing covering space, with no orientation-reversing tubes connecting the two types of spaces, as such tubes would increase the genus.

\section{$7 \quad$ Examples of Wilson loops}

We will now proceed to calculate the leading terms in the vacuum expectation values of some simple Wilson loops. For all the examples we consider here, we will assume that the Wilson loop is carrying the integer $k=1$, so that the observable we are measuring is the trace of the holonomy of the gauge field around the loop taken in the fundamental representation. We will first compute each VEV in a single sector of the theory, and then describe how it is different in the coupled theory. In general, given a Wilson loop $\gamma$ on the sphere, if 


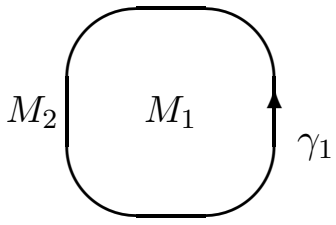

(a)

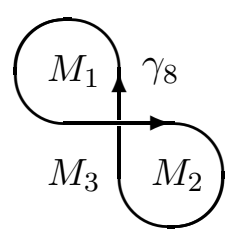

(b)

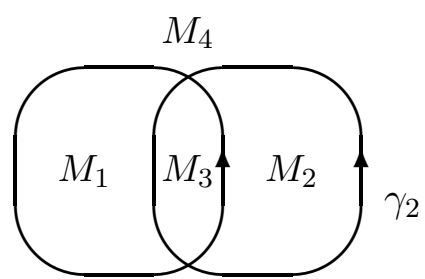

(c)

Figure 11: Wilson loops on the sphere

the area $A_{i}$ of a region $M_{i}$ is taken to infinity, the computation of $\left\langle W_{\gamma}\right\rangle$ is equivalent to computing the expectation value of $\gamma$ on the infinite plane. In this limit, the number of sheets in the coverings that contribute to the VEV is limited, since the number of sheets in the infinite region must be 0 . For such a Wilson loop, this allows us to compute the complete expectation value as a sum over a finite number of covering maps. The expectation values of Wilson loops on the plane have been previously considered by Kazakov and Kostov using loop equations derived by Makeenko and Migdal[14, [15]. Using these loop equation techniques, Kazakov was able to give a general algorithm for computing the VEV of an arbitrary Wilson loop in the plane[16]. N. Bralic also derived an algorithm for exact loop averages on the plane using a non-Abelian form of Stokes theorem[17]. In both of these works the gauge theory was taken to be $U(N)$. This corresponds to dropping the $U(1)$ term in the quadratic Casimir, and effectively removes handles and tubes from the theory, except those orientation-reversing tubes produced by $\Omega$-points. We compare the results computed using the string theory picture with the computations of Kazakov and Bralic, and verify that they are the same.

- $G=0, \gamma=\gamma_{1}, k=1$

We will first consider the trivial Wilson loop $\gamma=\gamma_{1}$ on the sphere (Figure 117a). The loop $\gamma$ divides the sphere into two regions $M_{1}, M_{2}$ of areas $A_{1}, A_{2}$. The first step in computing the expectation value of this Wilson loop in a single sector of the theory is to assign integers $I_{1}, I_{2}$ to the two regions, indicating how many $\Omega$-points are in each region. In this case, we have

$$
I_{1}=I_{2}=1
$$

We can now compute a set of relations between the numbers $n_{1}, n_{2}$ of sheets over the two 
regions for any fixed cover. We will assume that $M_{1}$ lies on the inside of $\gamma$. Since $k=1$, it follows that for any covering space $n_{1}=n_{2}+1$. The leading term in the expansion of $\left\langle W_{\gamma}\right\rangle_{+}$ is therefore proportional to $\exp \left(-\lambda A_{1} / 2\right)$, and corresponds to those covers that have a single sheet over $M_{1}$ and nothing over $M_{2}$. In fact, there is only a single such cover, except for possible handles, since there are no nontrivial permutations on a single sheet. Suppressing the functional dependence on $\lambda$ and $N$, the first contribution to $\left\langle W_{\gamma}\right\rangle_{+}$is given by

$$
W_{1}\left(A_{1}\right)=D_{1}\left(A_{1}\right)=e^{-\frac{\lambda A_{1}}{2}} \sum_{h} \frac{\left(\lambda A_{1}\right)^{h}}{2^{h} h !} N^{1-2 h}=N e^{-\frac{\lambda A_{1}}{2}+\frac{\lambda A_{1}}{2 N^{2}}} .
$$

The next set of covering spaces that contribute are those with $n_{1}=2, n_{2}=1$. By an argument practically identical to that leading to (6.8), the contribution from these covering spaces is given by

$$
\begin{aligned}
W_{2}\left(A_{1}, A_{2}\right) & \left.=D_{1}\left(A_{2}+2 A_{1}\right) \cdot \sum_{i, t} \frac{\left(\lambda A_{1}\right)^{2 i+t}}{(2 i) ! t !} N^{2-2 i-2 t}\left(1-\frac{\lambda A_{1}}{(2 i+1) N^{2}}\right)\right) . \\
& =N^{2} D_{1}\left(A_{2}+2 A_{1}\right) e^{\frac{\lambda A_{1}}{N^{2}}}\left[\cosh \frac{\lambda A_{1}}{N}-\frac{1}{N} \sinh \frac{\lambda A_{1}}{N}\right] \\
& =e^{-\frac{\lambda\left(A_{2}+2 A_{1}\right)}{2}}\left[N^{3}+N\left(\lambda A_{1}+\frac{\lambda A_{2}}{2}+\frac{1}{2}\left(\lambda A_{1}\right)^{2}\right)+\mathcal{O}\left(N^{-1}\right)\right] .
\end{aligned}
$$

This equation is slightly simpler then (6.8) because here we are working in the space of possibly disconnected covers, and there is only one $\Omega$-point in the region $M_{1}$.

We will now consider the expectation value in the full coupled theory. The relation between the numbers $n_{i}$ of orientation-preserving sheets and the numbers $\tilde{n}_{i}$ of orientationreversing sheets is given by

$$
n_{1}-\tilde{n}_{1}=n_{2}-\tilde{n}_{2}+1
$$

where $n_{1} \geq n_{2}$ and $\tilde{n}_{2} \geq \tilde{n}_{1}$. The leading terms in an expansion in the number of sheets are given by

$$
\begin{aligned}
&\left\langle W_{\gamma}\right\rangle= W_{1}\left(A_{1}\right)+W_{1}\left(A_{2}\right)+ \\
&+\sum_{\tilde{t}} \frac{(-\lambda)^{\tilde{t}}}{\tilde{t} !} N^{-2 \tilde{t}}\left(1-\frac{1}{N^{2}}\right)\left[A_{1}, A_{2}\right)+W_{2}\left(A_{2}+2 A_{1}\right) A_{1}^{\tilde{t}} \\
&\left.+D_{1}\left(2 A_{2}+A_{1}\right) A_{2}^{\tilde{t}}\right]+\mathcal{O}\left(e^{-\lambda\left(A_{1}+A_{2}\right)}\right)
\end{aligned}
$$

where the last term corresponds to the covers with $n_{1}=\tilde{n}_{1}=1$ and $n_{2}=\tilde{n}_{2}=1$, connected by an arbitrary number of orientation-reversing tubes. Note that the number of $\Omega$-points in each region is independent of which sector of the theory we are calculating in this example. 
Finally, we consider the VEV of the trivial Wilson loop when the area $A_{2}$ goes to infinity. Since the only covers that contribute in this limit are those with $n_{2}=\tilde{n}_{2}=0$, it follows that the only relevant covers are exactly those that gave rise to the leading term (7.2), and

$$
\lim _{A_{2} \rightarrow \infty}\left\langle W_{\gamma}\right\rangle=W_{1}\left(A_{1}\right)=N e^{-\frac{\lambda A_{1}}{2}} e^{\frac{\lambda A_{1}}{2 N^{2}}}
$$

Here we recognize the exact result of $\mathrm{QCD}_{2}$ for the expectation value of a non-intersecting Wilson loop on the infinite plane, $\left\langle W_{R}(A)\right\rangle=N^{n} \exp \left[-\lambda A C_{2}(R) / 2 N\right]$, for a loop in the fundamental representation where $C_{2}(\square)=N-1 / N$.

- $G=0, \gamma=\gamma_{8}, k=1$

We will next consider the figure- 8 Wilson loop $\gamma=\gamma_{8}$ on the sphere (Figure 11]b). This loop divides the sphere into 3 regions $M_{1}, M_{2}, M_{3}$ of areas $A_{1}, A_{2}, A_{3}$. This Wilson loop is equivalent to a Wilson loop winding twice around a point; as $A_{3} \rightarrow 0$, the VEV of this Wilson loop is equal to the VEV of $\gamma_{1}$ carrying the integer $k=2$ and the permutation $\sigma_{2}$. We will assume that $M_{1}$ lies on the inside of $\gamma$, and $M_{2}$ lies on the outside. In the orientation-preserving sector, the numbers of $\Omega$-points in the 3 regions are given by

$$
I_{1}=I_{2}=1, \quad I_{3}=0
$$

The relations between the numbers of sheets in the 3 regions are

$$
n_{1}=n_{3}+1=n_{2}+2 \text {. }
$$

In this sector, the leading term in the expansion of $\left\langle W_{\gamma}\right\rangle_{+}$is proportional to $\exp \left(-\lambda\left(2 A_{1}+\right.\right.$ $\left.A_{3}\right) / 2$ ). This term is the only one that survives in the limit $A_{2} \rightarrow \infty$, and is given by

$$
\begin{aligned}
\lim _{A_{2} \rightarrow \infty}\left\langle W_{\gamma}\right\rangle & =D_{1}\left(2 A_{1}+A_{3}\right) \cdot \sum_{i, t} \frac{\left(\lambda A_{1}\right)^{2 i+t}}{(2 i) ! t !} N^{-2 i-2 t}\left(1-\frac{\lambda A_{1}}{(2 i+1)}\right) \\
& =\frac{1}{2} D_{1}\left(2 A_{1}+A_{3}\right) \cdot e^{\frac{\lambda A_{1}}{N^{2}}}\left[(N+1) e^{-\frac{\lambda A_{1}}{N}}-(N-1) e^{\frac{\lambda A_{1}}{N}}\right] \\
& =e^{-\frac{\lambda\left(2 A_{1}+A_{3}\right)}{2}}\left[N\left(1-\lambda A_{1}\right)+\mathcal{O}\left(N^{0}\right)\right]
\end{aligned}
$$

The leading terms in this expansion correspond to maps from the disk to $S^{2}$ with a single branch point in the region $M_{1}$ arising either as a branch point or from the $\Omega$-point in that region. The expression (7.12) is identical to the result for the planar Wilson loop VEV computed by Kazakov in [16] and by Bralic in [17], if we neglect the terms arising from the different gauge group. 


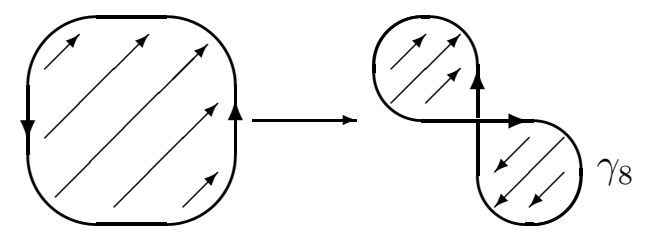

Figure 12: Map giving leading term in figure 8 VEV

In the coupled theory, the relations between the numbers of sheets are given by

$$
\begin{gathered}
n_{1}-\tilde{n}_{1}=n_{3}-\tilde{n}_{3}+1=n_{2}-\tilde{n}_{2}+2, \\
n_{1} \geq n_{3} \geq n_{2}, \quad \tilde{n}_{2} \geq \tilde{n}_{3} \geq \tilde{n}_{1} .
\end{gathered}
$$

The first set of covers that contribute are those with $n_{3}=\tilde{n}_{3}=0$ and $n_{1}=1, \tilde{n}_{2}=1$. This is also the only set of terms that survive in the limit $A_{3} \rightarrow \infty$. For these covers, as mentioned in in section 5 , we must take care with the placement of $\Omega$-points. The two edges of the graph of $\gamma$ contribute $\Omega^{-1}$-points to regions $M_{1}$ and $M_{2}$. The set of covers at the vertex is exactly that described explicitly in the first example at the end of section 5 (5.16). The analysis there indicated that the effect of the vertex was simply to allow the two sheets of opposite orientation to connect with a twist as part of the same sheet. The contributions from these covering spaces are thus given by

$$
\lim _{A_{3} \rightarrow \infty}\left\langle W_{\gamma}\right\rangle=D_{1}\left(A_{1}+A_{2}\right) .
$$

The leading term in this expansion corresponds to a map from the disk into $M_{1} \cup M_{2}$ which is twisted at the intersection point, and has no other singularities (see Figure 12). Again, this is exactly consistent with the results given by Kazakov and Bralic in [16], [17].

Note that in the limit $A_{3} \rightarrow \infty$ the VEV of this Wilson loop goes to 0 in a single sector, since there are no orientation-preserving covering maps with $\gamma$ as a boundary and with no sheets over $M_{3}$.

- $G=0, \gamma=\gamma_{2}, k=1$

We now consider the VEV of the pair of interlocking Wilson loops $\gamma=\gamma_{2}$ on the sphere (Figure 117). In a single sector, the regions $M_{1}, M_{2}, M_{3}, M_{4}$ with areas $A_{1}, A_{2}, A_{3}, A_{4}$ have the quantities of $\Omega$-points

$$
I_{1}=I_{2}=0, \quad I_{3}=I_{4}=1 .
$$


Assuming that region $M_{3}$ is inside of both curves, the numbers of sheets in each region satisfy

$$
n_{3}=n_{1}+1=n_{2}+1=n_{4}+2 .
$$

The term corresponding to the covers with $n_{3}=2$ is the only term in the coupled theory that survives in the limit $A_{4} \rightarrow \infty$, and is given by

$$
\begin{aligned}
\lim _{A_{4} \rightarrow \infty}\left\langle W_{\gamma}\right\rangle & \left.=D_{1}\left(A_{1}+A_{2}+2 A_{3}\right)\left[\sum_{i, t} \frac{\left(\lambda A_{3}\right)^{2 i+t}}{(2 i) ! t !} N^{1-2 i-2 t}\left(1-\frac{\lambda A_{3}}{(2 i+1) N^{2}}\right)\right)\right] \\
& =N D_{1}\left(A_{1}+A_{2}+2 A_{3}\right) e^{\frac{\lambda A_{3}}{N^{2}}}\left[\cosh \frac{\lambda A_{3}}{N}-\frac{1}{N} \sinh \frac{\lambda A_{3}}{N}\right] \\
& =e^{-\lambda\left(A_{1}+A_{2}+2 A_{3}\right)}\left[N^{2}+\frac{1}{2}\left(\lambda\left(A_{1}+A_{2}+2 A_{3}\right)+\left(\lambda A_{3}\right)^{2}\right)+\mathcal{O}\left(\frac{1}{N^{2}}\right)\right]
\end{aligned}
$$

in agreement with the calculation of Bralic [17].

In the coupled theory, we can enumerate the covers that contribute in the limit $A_{2} \rightarrow \infty$. There are two types of covers that are of interest, each with $n_{3}=1, \tilde{n}_{4}=1$; one class of covers has $n_{1}=\tilde{n}_{1}=0$, and the other class has $n_{1}=\tilde{n}_{1}=1$. The first class of maps has two vertex points with twists described by (5.16), and only has handles as possible singularities. The contribution from this class of maps is given by

$$
\frac{1}{N} D_{1}\left(A_{3}+A_{4}\right)
$$

The leading term here corresponds to a map from the annulus onto $M_{3} \cup M_{4}$ with twists at both intersection points. The second class of maps has $\Omega$-points distributed according to the distribution of sheets by the same rules as in a single sector. The numbers of $\Omega$-points for such covering maps are

$$
I_{1}=I_{2}=1, \quad I_{3}=I_{4}=0
$$

The contribution from maps of this type is

$$
D_{1}\left(A_{1}+A_{3}\right) D_{1}\left(A_{1}+A_{4}\right)\left(1-\frac{1}{N^{2}}\right) \sum_{\tilde{t}} \frac{\left(-\lambda A_{1}\right)^{\tilde{t}}}{\tilde{t} !} N^{-2 \tilde{t}} .
$$

The leading term in this contribution arises from a disconnected pair of sheets; an orientationpreserving sheet over $M_{1}$ and $M_{3}$, and an orientation-reversing sheet over $M_{2}$ and $M_{4}$. The total VEV of the interlocked loops in the limit $A_{2} \rightarrow \infty$ is thus given by

$$
\begin{aligned}
\lim _{A_{2} \rightarrow \infty}\left\langle W_{\gamma}\right\rangle & =\frac{1}{N} D_{1}\left(A_{3}+A_{4}\right)+N D_{1}\left(2 A_{1}+A_{3}+A_{4}\right)\left(1-\frac{1}{N^{2}}\right) e^{-\frac{\lambda A_{1}}{N^{2}}} \\
& =N^{2}+\left(\frac{A_{3}+A_{4}}{2}\right)+\mathcal{O}\left(N^{-2}\right) .
\end{aligned}
$$




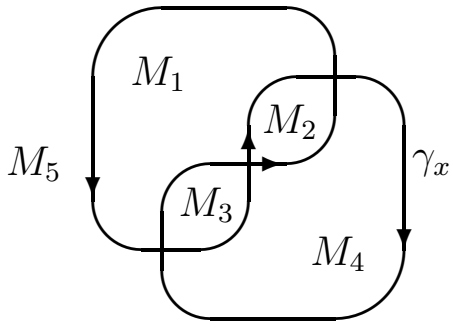

Figure 13: Another Wilson loop, $\gamma_{x}$

Neglecting the extra $U(1)$ terms, this is in agreement with Kazakov's results in 16.

- $G=0, \gamma=\gamma_{x}, k=1$

We will consider one more Wilson loop on the sphere, the loop $\gamma=\gamma_{x}$ shown in Figure 13 . We will only consider the VEV of this Wilson loop in the planar limit as $A_{5} \rightarrow \infty$. In this limit, there are no orientation-preserving covering maps; however, there are 4 classes of coverings which contribute to the $\mathrm{VEV}$ in the full theory. In all the relevant cases, the coverings of $M_{1}$ and $M_{4}$ consist of a single orientation-preserving sheet and a single orientation-reversing sheet, respectively. The numbers of sheets in regions 2 and 3 satisfy $n_{2}=\tilde{n}_{2} \leq 1, n_{3}=\tilde{n}_{3} \leq 1$. The four possible cases are described by taking $\left(n_{2}, n_{3}\right)=$ $(0,0),(0,1),(1,0),(1,1)$.

In the first case, $\left(n_{2}, n_{3}\right)=(0,0)$, all three vertices are of the type described by (5.16). Only handle singularities are allowed, so the contribution from this case to the VEV is given by

$$
\frac{1}{N^{2}} D_{1}\left(A_{1}+A_{4}\right)
$$

where the leading term $1 / N$ arises from a map from a torus with a single boundary component onto regions $M_{1}, M_{4}$ with twists at all vertices.

In the cases $\left(n_{2}, n_{3}\right)=(0,1),\left(n_{2}, n_{3}\right)=(1,0)$, one of the two vertices is of the type described by (5.16). The other two vertices have $\Omega$-points associated with an adjacent region with two sheets $\left(M_{3}, M_{2}\right.$ respectively). The contributions from these two classes of maps are

$$
\begin{aligned}
& D_{1}\left(A_{1}+2 A_{3}+A_{4}\right)\left(1-\frac{1}{N^{2}}\right) \sum_{\tilde{t}} \frac{\left(-\lambda A_{3}\right)^{\tilde{t}}}{\tilde{t} !} N^{-2 \tilde{t}}, \\
& D_{1}\left(A_{1}+2 A_{2}+A_{4}\right)\left(1-\frac{1}{N^{2}}\right) \sum_{\tilde{t}} \frac{\left(-\lambda A_{2}\right)^{\tilde{t}}}{\tilde{t} !} N^{-2 \tilde{t}},
\end{aligned}
$$




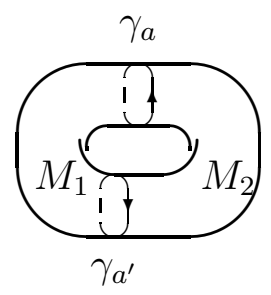

Figure 14: Wilson loops on a torus

respectively.

Finally, we consider the case $\left(n_{2}, n_{3}\right)=(1,1)$. In this case, the $\Omega$-points from the outer vertices can be associated with regions $M_{2}, M_{3}$. The central vertex, however, is of the type described by (5.22), and carries a factor of $-N\left(1-1 / N^{2}\right)$. The contribution from this class of coverings is thus given by

$$
-D_{1}\left(A_{1}+2 A_{2}+2 A_{3}+A_{4}\right)\left(1-\frac{1}{N^{2}}\right) \sum_{\tilde{t}} \frac{\left(-\lambda\left(A_{2}+A_{3}\right)\right)^{\tilde{t}}}{\tilde{t} !} N^{-2 \tilde{t}} .
$$

The total VEV of $\gamma$ in the limit $A_{5} \rightarrow \infty$ is thus given by

$$
\begin{aligned}
\lim _{A_{5} \rightarrow \infty}\left\langle W_{\gamma}\right\rangle= & \frac{1}{N^{2}} D_{1}\left(A_{1}+A_{4}\right)+D_{1}\left(A_{1}+2 A_{3}+A_{4}\right)\left(1-\frac{1}{N^{2}}\right) e^{-\frac{\lambda A_{3}}{N^{2}}} \\
& +D_{1}\left(A_{1}+2 A_{2}+A_{4}\right)\left(1-\frac{1}{N^{2}}\right) e^{-\frac{\lambda A_{2}}{N^{2}}} \\
& -D_{1}\left(A_{1}+2 A_{2}+2 A_{3}+A_{4}\right)\left(1-\frac{1}{N^{2}}\right) e^{-\frac{\lambda\left(A_{2}+A_{3}\right)}{N^{2}}} \\
= & N+\frac{A_{1}+A_{4}}{2 N}+\mathcal{O}\left(N^{-3}\right) .
\end{aligned}
$$

This agrees again with Kazakov's results [16] when $U(1)$ terms are neglected.

- $G=1, \gamma=\gamma_{a} \cup \gamma_{a^{\prime}}, k=1$

Finally, we will consider an interesting Wilson loop on the torus. If we consider a single Wilson loop $\gamma_{a}$ on the torus that is homologically nontrivial (see Figure 14), clearly the VEV of that Wilson loop must be 0 , since the number of sheets of any cover must differ by 1 across the loop, and the same region is on both sides of the loop. The partition function on the torus is physically equivalent to the finite temperature partition function in flat space. In the finite temperature case we would regard the length of one cycle as the spatial extent of the world and the other as $1 / \beta$, where $\beta=k$. Temperature. The loop can be thought of as the 
Polyakov loop, which is the order parameter for the finite temperature phase transition. The fact that it always vanishes simply means that in $\mathrm{QCD}_{2}$ there is no deconfinement transition, no matter how large the temperature is.

On the other hand, if we consider a Wilson loop composed of two disjoint Wilson loops $\gamma=\gamma_{a} \cup \gamma_{a^{\prime}}$ the VEV can be nontrivial as long as the loops $\gamma_{a}$ and $\gamma_{a^{\prime}}$ are inverses in homology. If the regions into which the torus is divided are $M_{1}, M_{2}$ then the leading terms in the coupled theory are given by

$$
\left\langle W_{\gamma}\right\rangle=\frac{1}{N}\left(D\left(A_{1}\right)+D\left(A_{2}\right)\right)+\mathcal{O}\left(e^{-\frac{\lambda\left(A_{1}+A_{2}\right)}{2}}\right)
$$

where the leading terms are given by maps from the annulus into one of the two regions bounded by $\gamma$. The structure of this VEV can be used to analyze the spectrum of heavy quark mesons in the theory.

\section{Dynamical quarks}

So far we have described pure $\mathrm{QCD}_{2}$, including an arbitrary number of Wilson loops, in terms of a geometrical sum of maps-a string theory. This allows us to discuss infinitely heavy quarks in terms of a simple open string theory. More interesting is to consider the theory with dynamical quarks. This should be describable in terms of an open string theory with dynamical boundaries.

In order to relate the full theory to a string theory start with the partition function for QCD with, for simplicity, a single Dirac fermion in the fundamental representation of $S U(N)$,

$$
Z=\int \mathcal{D} \bar{\Psi} \mathcal{D} \Psi \mathcal{D} A_{\mu} e^{\int d^{2} x\left[\bar{\Psi} \gamma^{\mu}\left(\partial_{\mu}-\mathbf{A}_{\mu}\right) \Psi-m \bar{\Psi} \Psi-\frac{1}{4 \tilde{g}^{2}} \operatorname{Tr} F^{\mu \nu} F_{\mu \nu}\right]}
$$

Integrating out the quarks one is left with

$$
Z=\int \mathcal{D} A_{\mu} e^{-\frac{1}{4 \tilde{g}^{2}} \int d^{2} x\left[\operatorname{Tr} F^{\mu \nu} F_{\mu \nu}\right]+\operatorname{Tr} \ln \left[\gamma^{\mu}\left(\partial_{\mu}-\mathbf{A}_{\mu}\right)-m\right]}
$$

One can then rewrite the logarithm of the fermion determinant in terms of a sum over paths using standard techniques. One expresses the determinant as an integral over proper time $(T)$ of the exponential of the Hamiltonian $H=\gamma^{\mu} D_{\mu}-m$, where $D_{\mu}$ is the covariant derivative in the background gauge field. The usual Hamiltonian path integral representation 
follows,

$$
\begin{aligned}
S_{\Psi}(A) & \equiv \operatorname{Tr} \ln \left[\gamma^{\mu} D_{\mu}-m\right]=\operatorname{Tr} \int_{0}^{\infty} \frac{d T}{T} e^{i T\left(\gamma^{\mu} D_{\mu}-m\right)} \\
& =\int_{0}^{\infty} \frac{d T}{T} \oint_{x^{\mu}(0)=x^{\mu}(T)} \mathcal{D} x^{\mu}(t) \int \mathcal{D} p^{\mu}(t) \operatorname{tr} P\left\{e^{-i \int_{o}^{T}\left(p^{\mu} \dot{x}_{\mu}-\gamma^{\mu} p_{\mu}+\gamma^{\mu} \mathbf{A}_{\mu}+m\right) d t}\right\},
\end{aligned}
$$

where the $P$ denotes path ordering of the Dirac matrices as well as of the gauge field $\left(\mathbf{A}_{\mu}\right)$, which is a matrix in the fundamental representation. After a shift $\left(p_{\mu} \rightarrow p_{\mu}+A_{\mu}\right)$, we have,

$$
S_{\Psi}(A)=\int_{0}^{\infty} \frac{d T}{T} \oint_{x^{\mu}(0)=x^{\mu}(T)} \mathcal{D} x^{\mu}(t) W_{x}(A) \int \mathcal{D} p^{\mu}(t) \operatorname{tr} P\left\{e^{-i \int_{o}^{T}\left(p^{\mu} \dot{x}_{\mu}-\gamma^{\mu} p_{\mu}+m\right) d t}\right\},
$$

where $W_{x}(A)=\operatorname{Tr} P \exp \oint d x^{\mu} \mathbf{A}_{\mu}(\mathbf{x})$ is the Wilson loop associated with the quark path.

This approach was studied by Strominger in 1981 [20]. He showed that in two dimensions the path integral over the momentum $p^{\mu}$ can be easily evaluated, and that it simply yields a factor of $(-1)^{\omega_{x}} \exp (-i m T)$, where $\omega_{x}$ is the winding number of the loop, namely the number of $2 \pi$ rotations the quark makes around the closed path. Thus, following Strominger, we see that the $\mathrm{QCD}_{2}$ partition function can be written as

$$
Z=\sum_{n} \frac{1}{n !} \prod_{i=1}^{n}\left\{\int_{0}^{\infty} \frac{d T_{i}}{T_{i}} \oint_{x_{i}^{\mu}(0)=x_{i}^{\mu}(T)} \mathcal{D} x_{i}^{\mu}(-1)^{\omega_{x_{i}}} e^{-i m T_{i}}\right\}\left\langle\prod_{i=1}^{n} W_{x_{i}}(A)\right\rangle
$$

We see that the partition function can be written as a weighted sum over closed quark paths, times the expectation value of the Wilson loops in the fundamental representation for each path. The latter term can be expressed, following this paper, as a sum of open string maps. (There is, of course, always the trivial term with no quarks, which corresponds to closed string maps). We therefore can regard the complete partition function as being given by an open plus a closed string theory, whose boundaries are dynamical.

If we could find the world sheet string Lagrangian, $\mathcal{L}_{s}$, that describes the set of maps derived in this paper, we could rewrite the QCD partition function completely in terms of this string theory; one of the novel features of this theory would be the fact that the boundaries of the open string have dynamics of their own.

A challenge for this approach to QCD is to rederive the meson spectrum calculated by perturbation theory techniques in the large $N$ limit by 't Hooft [18], [19]. The meson spectrum is calculated by finding the poles in correlators of quark bilinears. These too have an obvious representation as sums over quark paths,

$$
\langle\bar{\Psi} \Psi(x) \bar{\Psi} \Psi(y)\rangle=\int d T \oint_{x, y} \mathcal{D} x^{\mu}(-1)^{\omega_{x y}} e^{-i m T}\left\langle W_{x y}(A)\right\rangle
$$


where the sum is over all loops that pass through both $x$ and $y$ in proper time $T$ and, since $N \rightarrow \infty$, we drop all other quark loops. Strominger [20], as well as Bars and Hanson [21], argued that this will reproduce the 't Hooft spectrum. The argument was that in the infinite momentum frame quarks cannot backtrack. Thus the quark loops are simple and yield an area law corresponding to a linear potential. This produces the 't Hooft spectrum. However this is not really a string argument. It remains a challenge to derive the 't Hooft spectrum using the above, manifestly gauge and Lorentz invariant, formula for the meson correlator, where one inserts the large $N$ expressions for the Wilson loops. This is the procedure that one would follow to calculate the meson spectrum in the four dimensional string theory of QCD, once the theory has been constructed.

\section{Conclusions}

The primary conclusion of this paper and of our previous work is that two-dimensional QCD is equivalent to a string theory. We have shown that the partition function and the expectation values of Wilson loops on arbitrary space-time manifolds can be derived in terms of a sum of maps of a two-dimensional world sheet. We have given the precise set of rules that determine the nature of the maps and the weights that appear in their sum. These rules define the $\mathrm{QCD}_{2}$ string theory. However, we still do not know a path integral formulation that would produce this string theory. In some ways our definition of the QCD string theory as a sum of maps is more explicit than a specification of the string Lagrangian. If one possessed a string Lagrangian, one would then be faced with the problem of how to perform the path integral to calculate physical observables. Indeed, one would be happy to prove that the path integral was equivalent to a simple set of maps with well defined weights. However, if explicit calculations were our goal, we might as well stick with QCD in its field theoretic formulation, where the calculations are even simpler. Our goals are different. They are first, to verify that in the simplest of cases QCD can be described by a string theory, and then to continue the two-dimensional string theory to four dimensions.

We have succeeded in the first goal- $\mathrm{QCD}_{2}$ is describable by a string theory. However, the present formulation of the theory does not allow us to continue to higher dimension. The rules for constructing the maps that we have presented are inherently two-dimensional and do not have a straightforward extension to higher dimensions. The most important feature of the maps is that they exclude all finite folds. This feature of the string theory, 
we believe, is responsible for the absence of all particles in the closed string sector and for the simplicity of the resulting maps. In higher dimensions, however, folds can be removed by an arbitrarily small perturbation of the string map, and are presumably irrelevant to the theory. Possibly the dynamics of the QCD string that eliminates folds in two dimensions suppresses maps with extrinsic curvature in higher dimensions. We conjecture that this feature is responsible for the absence of the tachyon, the dilaton and the graviton. But it is hard to guess the appropriate formulation of three or four dimensional string theory with our present understanding. It seems that a necessary intermediate step is the construction of the first quantized string field theory that yields our maps.

In constructing the $\mathrm{QCD}_{2}$ Lagrangian we should be guided by the properties of the maps that we have discovered. The weight of our maps is the exponential of the area, suggesting that the string action contains the Nambu-Goto term. Perhaps the most important other feature of the maps is the suppression of folds of finite size. This suggests that the string action contain terms that suppress maps with extrinsic curvature. Here we might try to draw some lessons from the recent numerical simulations of higher dimensional string theories. Other significant clues are the minus signs associated with branchpoints and with orientation reversing tubes. This suggests that the string theory be formulated with some kind of fermionic variables in addition to the bosonic string coordinates. Finally, the $\Omega$ points that occur on target space manifolds of genus not equal to one and when Wilson loops are present should provide strong constraints on the string Lagrangian. The fact that the number of $\Omega$-points is equal to the Euler characteristic of the surface is an indication that these objects are related to global structures on the manifold.

In addition to constructing the string action there remains much interesting work to be done in two dimensions. As we discussed above, it would be very instructive to reproduce the meson spectrum of $\mathrm{QCD}_{2}$ (with quarks) by stringy quarks. It would also be instructive to reproduce the spectrum of baryons, which should be treated as solitons in the string theory [22].

\section{A Generalized Frobenius relations}

In this appendix we we generalize the well known Frobenius relations (1.6) to the case of the composite representations $\bar{S} R$. First let us establish some notations. A conjugacy class of permutations in the symmetric group $S_{n}$ on $n$ objects is determined by the number of cycles 
of each length in a representative permutation; all permutations in a conjugacy class have the same set of cycle lengths. For a given permutation $\sigma \in S_{n}$, we denote the total number of cycles in the permutation by $K_{\sigma}$. Given a permutation $\sigma$ with cycles of length $k_{1}, \ldots, k_{K_{\sigma}}$, we denote the conjugacy class of $\sigma$ by $T_{\sigma}$. We will identify the conjugacy class $T_{\sigma}$ with the associated set of cycle lengths $\left\{k_{1}, \ldots, k_{K_{\sigma}}\right\}$. Thus, we can apply the set theoretic operations $\cup, \cap, \subset$ to conjugacy classes. We will denote by $\sigma_{k}$ the number of cycles in $\sigma$ of length $k$. A standard notation for a permutation is $\sigma=\left[1^{\sigma_{1}} \ldots i^{\sigma_{i}} \ldots n^{\sigma_{n}}\right]$, where $\sigma_{1}+2 \sigma_{2}+\ldots n \sigma_{n}=n$. We denote the order of the conjugacy class of $\sigma$ by $\left|T_{\sigma}\right|$, and the number of elements of $S_{n}$ that commute with $\sigma$ by

$$
C_{\sigma}=n ! /\left|T_{\sigma}\right|=\prod_{i=1}^{n} i^{\sigma_{i}} \sigma_{i} ! .
$$

We will abuse notation slightly by sometimes using conjugacy classes in place of permutations, where an equation depends only on the conjugacy class of a permutation.

The standard Frobenius relations are

$$
\begin{aligned}
& \chi_{R}(U)=\sum_{\sigma \in S_{k}} \frac{\chi_{R}(\sigma)}{k !} \Upsilon_{\sigma}(U), \\
& \Upsilon_{\sigma}(U)=\sum_{R \in Y_{k}} \chi_{R}(\sigma) \chi_{R}(U),
\end{aligned}
$$

where we recall that

$$
\Upsilon_{\sigma}(U)=\prod_{i=1}^{K_{\sigma}}\left(\operatorname{Tr} U^{k_{i}}\right) .
$$

We wish to derive a more general set of relations

$$
\begin{aligned}
\chi_{\bar{S} R}(U) & =\sum_{\sigma, \tau} \frac{\chi_{R}(\sigma) \chi_{S}(\tau)}{n ! \tilde{n} !} \Upsilon_{\bar{\tau} \sigma}\left(U, U^{\dagger}\right), \\
\Upsilon_{\bar{\tau} \sigma}\left(U, U^{\dagger}\right) & =\sum_{R, S} \chi_{R}(\sigma) \chi_{S}(\tau) \chi_{\bar{S} R}(U) .
\end{aligned}
$$

We will take these equations to define the class functions $\Upsilon_{\bar{\tau} \sigma}\left(U, U^{\dagger}\right)$. Clearly, for any $\sigma, \tau$ the function $\Upsilon_{\bar{\tau} \sigma}\left(U, U^{\dagger}\right)$ can be written as a sum of terms proportional to products of the form $\Upsilon_{\sigma^{\prime}}(U) \Upsilon_{\tau^{\prime}}\left(U^{\dagger}\right)$, with a leading term of $\Upsilon_{\sigma}(U) \Upsilon_{\tau}\left(U^{\dagger}\right)$. From (A.5) and the orthonormality of the characters, $\int d U \chi_{\bar{S} R}(U) \chi_{\bar{S}^{\prime} R^{\prime}}\left(U^{\dagger}\right)=\delta_{R, R^{\prime}} \delta_{S, S^{\prime}}$, it follows that

$$
\int d U \Upsilon_{\bar{\tau} \sigma}\left(U, U^{\dagger}\right) \Upsilon_{\bar{\tau}^{\prime} \sigma^{\prime}}\left(U, U^{\dagger}\right)=\delta_{T_{\sigma}, T_{\tau^{\prime}}} \delta_{T_{\sigma^{\prime}}, T_{\tau}} C_{\sigma} C_{\tau}
$$

Thus, in order to construct the functions $\Upsilon_{\bar{\tau} \sigma}\left(U, U^{\dagger}\right)$, it will suffice to orthonormalize the set of functions $\Upsilon_{\sigma}(U) \Upsilon_{\tau}\left(U^{\dagger}\right)$. 
We claim that in fact

$$
\begin{aligned}
\Upsilon_{\bar{\tau} \sigma}\left(U, U^{\dagger}\right) & =\sum_{v \subset T_{\sigma}} \sum_{v^{\prime} \subset T_{\tau}, v \approx v^{\prime}}(-1)^{K_{v}} C_{v} \Upsilon_{\sigma \backslash v}(U) \Upsilon_{\tau \backslash v}\left(U^{\dagger}\right) \\
& =\Upsilon_{\sigma}(U) \Upsilon_{\tau}\left(U^{\dagger}\right)+\cdots
\end{aligned}
$$

where $v$ and $v^{\prime}$ are summed over all subsets of the sets $\left\{k_{1}, \ldots, k_{K_{\sigma}}\right\}$ and $\left\{k_{1}^{\prime}, \ldots, k_{K_{\tau}}^{\prime}\right\}$ that define $T_{\sigma}, T_{\tau}$. Thus $\sigma \backslash v$ refers to the class of permutations whose cycles have lengths belonging to the set $\left\{k_{1}, \ldots, k_{K_{\sigma}}\right\}$ minus the set $v$. We will take (A.7) as the definition of $\Upsilon_{\bar{\tau} \sigma}\left(U, U^{\dagger}\right)$, and prove that (A.6) is satisfied.

Every permutation $\sigma$ can be rewritten as a product of permutations $\sigma^{(l)}$ all of whose cycles are of length $l$. Formally, we write $\sigma=\prod \sigma^{(l)}$, where $\sigma^{(l)}=\left[l^{\sigma_{l}}\right]$. The functions $K_{\sigma}$ and $C_{\sigma}$, defined above, clearly satisfy

$$
\begin{aligned}
& K_{\sigma}=\sum_{l} K_{\sigma^{(l)}} \\
& C_{\sigma}=\prod_{l} C_{\sigma^{(l)}} .
\end{aligned}
$$

The functions $\Upsilon_{\sigma}$ can be written as $\Upsilon_{\sigma}=\prod_{l} \Upsilon_{\sigma^{(l)}}$. Similarly, if $v \subset T_{\sigma}$, then the set $\sigma \backslash v$ can also be decomposed as $\sigma \backslash v=\prod_{l}(\sigma \backslash v)^{(l)}=\prod_{l}\left(\sigma^{(l)} \backslash v^{(l)}\right)$.

From the Frobenius relations, we know that

$$
\int d U \Upsilon_{\sigma}(U) \Upsilon_{\tau}\left(U^{\dagger}\right)=\delta_{T_{\sigma}, T_{\tau}} C_{\sigma}=\prod_{l} \delta_{\sigma^{(l)}, \tau^{(l)}} C_{\sigma^{(l)}}=\prod_{l} \int d U_{l} \Upsilon_{\sigma^{(l)}}\left(U_{l}\right) \Upsilon_{\tau^{(l)}}\left(U_{l}^{\dagger}\right) .
$$

In other words, in any group integration over products of traces of $U, U^{\dagger}$, the product over cycles of different lengths can be taken outside the integral.

We therefore expect that $\Upsilon_{\bar{\tau} \sigma}\left(U, U^{\dagger}\right)$ can also be written as a product over cycles of equal length, namely

$$
\Upsilon_{\bar{\tau} \sigma}\left(U, U^{\dagger}\right)=\prod_{l} \Upsilon_{\overline{\tau^{(l)}} \sigma(l)}\left(U, U^{\dagger}\right) .
$$

The Ansatz, (A.7), is equivalent to the assertion that

$$
\Upsilon_{\overline{\tau^{(l)}} \sigma^{(l)}}\left(U, U^{\dagger}\right)=\sum_{k=0}^{\min \left(\sigma_{l}, \tau_{l}\right)}\left(\begin{array}{l}
\sigma_{l} \\
k
\end{array}\right)\left(\begin{array}{l}
\tau_{l} \\
k
\end{array}\right)(-1)^{k} l^{k} k !\left(\operatorname{Tr} U^{l}\right)^{\sigma_{l}-k}\left(\operatorname{Tr} U^{\dagger l}\right)^{\tau_{l}-k} .
$$

It is a consequence of (A.11) that

$$
\int d U \Upsilon_{\bar{\tau} \sigma}\left(U, U^{\dagger}\right) \Upsilon_{\bar{\tau}^{\prime} \sigma^{\prime}}\left(U, U^{\dagger}\right)=\prod_{l} \int d U_{l} \Upsilon_{{\overline{\tau^{(l)}}}^{(l)}}\left(U_{l}, U_{l}^{\dagger}\right) \Upsilon_{{\overline{\tau^{(l)}}}^{\prime} \sigma^{(l)^{\prime}}}\left(U_{l}, U_{l}^{\dagger}\right) .
$$


Therefore, our new basis will be orthogonal if we can prove that the $\Upsilon \overline{\tau^{(l)}} \sigma^{(l)}\left(U, U^{\dagger}\right)$ are orthogonal. Assume then, that we have permutations $\sigma, \tau, \sigma^{\prime}, \tau^{\prime}$ containing $s, t, s^{\prime}, t^{\prime}$ cycles of length $l$. Without loss of generality, we can assume that $s+t \geq s^{\prime}+t^{\prime}$, and $s \geq t$. In order to prove (A.6) for these permutations, it will suffice to prove that

$$
\int d U \Upsilon_{\bar{\tau} \sigma}\left(U, U^{\dagger}\right) \Upsilon_{\sigma^{\prime}}(U) \Upsilon_{\tau^{\prime}}\left(U^{\dagger}\right)=\delta_{s, t^{\prime}} \delta_{s^{\prime}, t} t^{s+t} s ! t !
$$

It is clear from counting factors of $U$ and $U^{\dagger}$ that the integral on the left of (A.15) is 0 unless $s+s^{\prime}=t+t^{\prime}$. Thus, if we define $r=s-t$, we have $t^{\prime}=s^{\prime}+r \leq s, s^{\prime} \leq t$. We can compute (A.15) directly from the definition (A.7),

$$
\begin{aligned}
\int & d U \Upsilon_{\bar{\tau} \sigma}\left(U, U^{\dagger}\right) \Upsilon_{\sigma^{\prime}}(U) \Upsilon_{\tau^{\prime}}\left(U^{\dagger}\right) \\
= & \sum_{i=0}^{t}\left(\begin{array}{c}
s \\
i
\end{array}\right)\left(\begin{array}{c}
t \\
i
\end{array}\right)(-1)^{i} l^{i} i !\left[l^{s+s^{\prime}-i}\left(s+s^{\prime}-i\right) !\right] \\
= & \sum_{i=0}^{t} \frac{s ! t !\left(s+s^{\prime}-i\right) !(-1)^{i} l^{s+s^{\prime}}}{(s-i) ! i !(t-i) !} \\
= & \left.l^{s+s^{\prime}} \frac{d^{s^{\prime}}}{d x^{s^{\prime}}}\right|_{x=1} s ! x^{r+s^{\prime}}(x-1)^{t}=l^{s+s^{\prime}} \delta_{t, s^{\prime}} s ! t !,
\end{aligned}
$$

where in (A.16) the sum over $i$ runs over the sets $v$ that contain $i$ cycles of length $l$, chosen in $\left(\begin{array}{l}s \\ i\end{array}\right)$ different ways. Thus, we have proven the assertion (A.15). It follows that (A.6) holds for all permutations, and thus that (A.7) correctly defines the functions for the generalized Frobenius relations.

We can also invert this relation and express the products $\Upsilon_{\sigma^{\prime}}(U) \Upsilon_{\tau^{\prime}}\left(U^{\dagger}\right)$ in terms of the $\Upsilon_{\bar{\tau} \sigma}\left(U, U^{\dagger}\right)$. Remarkably, the inverse formula has the same form (except for the absence of the $(-1)^{K_{v}}$ factor),

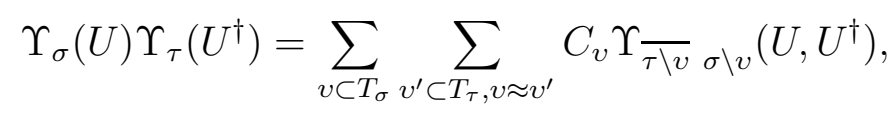

This can be proved by using the inverse for the terms with cycles of equal length,

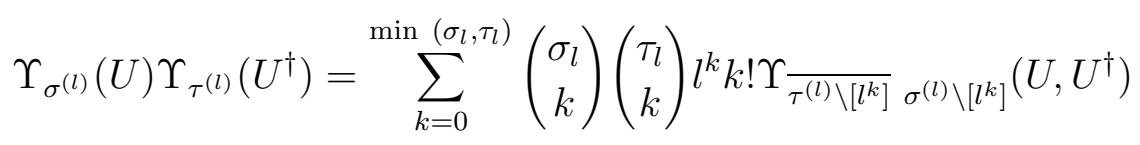

We give some examples of this orthonormal basis:

$$
\Upsilon_{\overline{[1]}[1]}\left(U, U^{\dagger}\right)=\operatorname{Tr}(U) \operatorname{Tr}\left(U^{\dagger}\right)-1
$$




$$
\begin{aligned}
\Upsilon_{\overline{\left[1^{3}\right]\left[1^{1}, 2^{1}\right]}}\left(U, U^{\dagger}\right) & =\operatorname{Tr}\left(U^{2}\right) \operatorname{Tr}(U) \operatorname{Tr}\left(U^{\dagger}\right)^{3}-3 \operatorname{Tr}\left(U^{2}\right) \operatorname{Tr}\left(U^{\dagger}\right)^{2} \\
\Upsilon_{\overline{\left[1^{n}\right]\left[1^{n}\right]}}\left(U, U^{\dagger}\right) & =\operatorname{Tr}(U)^{n} \operatorname{Tr}\left(U^{\dagger}\right)^{n}+\sum_{k=1}^{n}\left(\begin{array}{l}
n \\
k
\end{array}\right)^{2}(-1)^{k} k ! \operatorname{Tr}(U)^{n-k} \operatorname{Tr}\left(U^{\dagger}\right)^{n-k} \\
\Upsilon_{\overline{[n]}[n]}\left(U, U^{\dagger}\right) & =\operatorname{Tr}\left(U^{n}\right) \operatorname{Tr}\left(U^{\dagger n}\right)-n .
\end{aligned}
$$

A direct result of (A.7) is a useful relation for the dimensions of composite representations,

$$
\operatorname{dim} \bar{S} R=\sum_{\sigma, \tau} \frac{\chi_{R}(\sigma) \chi_{S}(\tau)}{n ! \tilde{n} !} \sum_{v \subset T_{\sigma}} \sum_{v^{\prime} \subset T_{\tau}, v \approx v^{\prime}}(-1)^{K_{v}} C_{v} N^{K_{\sigma \backslash v}} N^{K_{\tau \backslash v}} .
$$

Alternatively, if we use the product form,

$$
\operatorname{dim} \bar{S} R=\sum_{\sigma, \tau} \frac{\chi_{R}(\sigma) \chi_{S}(\tau)}{n ! \tilde{n} !} N^{K_{\sigma}+K_{\tau}} \prod_{l} F_{\sigma^{(l)}, \tau^{(l)}}\left(\frac{l}{N^{2}}\right)
$$

where

$$
F_{\sigma, \tau}(x) \equiv \sum_{v}(-1)^{v} v !\left(\begin{array}{l}
\sigma \\
v
\end{array}\right)\left(\begin{array}{l}
\tau \\
v
\end{array}\right) x^{v}=\left.\left(1-x \frac{d}{d y}\right)^{\tau} y^{\sigma}\right|_{y=1}
$$

These formulas are appropriate for a $1 / N$ expansion of the dimensions.

\section{Acknowledgements}

We would like to thank Orlando Alvarez for helpful discussions.

\section{References}

[1] A. Migdal, Zh. Eksp. Teor. Fiz. 69 , 810 (1975) (Sov. Phys. JETP. 42 413)

[2] B. Rusakov, Mod. Phys. Lett. A5, 693 (1990).

[3] D. Gross, preprint PUPT 1356, LBL 33415, HEPTH/9212149, December 1992.

[4] D. Gross, W. Taylor, preprint PUPT 1376, LBL 33458, UCB-PTH-93/02, HEPTH/9301068, January 1993.

[5] J. Minahan, preprint UVA-HET-92-10, HEPTH/9301003, January 1993.

[6] E. Witten, Comm. Math. Phys. 141,153 (1991).

[7] S. Samuel, Jour. Math. Phys. 21 , 2695 (1980). 
[8] C. Korthals-Altes, in Proc. 1974 Marseille Conf.; G. 't Hooft and B. deWit, Phys. Lett. 69B, 61 (1977); I. Bars and F. Green, Phys. Rev. D20, 3311 (1979); D. Weingarten, Phys. Lett. 90B, 285 (1980); D. Foerster, Nucl. Phys. B170(FS1), 107 (1980); A. Migdal, Phys. Rep. 102, 316 (1983); J. Droufe and J. Zuber, Phys. Lett 144B, 407 (1982).

[9] V. Kazakov, Phys. Lett. 128B, 316 (1983).

[10] I. Kostov, Phys. Lett. 138B, 191 (1984).

[11] I. Kostov, Nucl. Phys. B265, 223 (1986).

[12] K. H. O' Brien and J.-B. Zuber, Nucl. Phys. B253, 621 (1985), Phys. Lett. 144B, 407 (1984).

[13] D. Gross and E. Witten, Phys. Rev. D21, 446(1980).

[14] V. Kazakov and I. Kostov, Nucl. Phys. B176, 199 (1980).

[15] Y. Makeenko and A. Migdal, Phys. Lett. 88B, 135 (1979).

[16] V. Kazakov, Nucl. Phys. B179, 283 (1981).

[17] N. Bralic, Phys. Rev. D22, 3090 (1980).

[18] G. 't Hooft, Nucl. Phys. B72, 461 (1974).

[19] C. Callan, N. Coote and D. Gross, Phys. Rev. D13, 1649 (1976).

[20] A. Strominger, Phys. Lett. 101B, 271 (1981).

[21] I. Bars and A. Hanson, Phys. Rev. D13, 1744 (1976), Nucl. Phys. B111, 413 (1976).

[22] V. Kazakov and A. Migdal, Phys. Lett. 103B, 214 (1981). 